\title{
Espaços exponencialmente completos
}

\author{
Renato Leme Martin
}

TESE APRESENTADA

$\mathrm{AO}$

INSTITUTO DE MATEMÁTICA E ESTATÍSTICA

DA

UNIVERSIDADE DE SÃO PAULO

PARA

OBTENÇÃO DO GRAU DE MESTRE

EM

MATEMÁTICA

\section{Área de Concentração: Matemática Orientadora: Prof $\underline{a}$ Dr $\underline{\text { a }}$ Ofélia Teresa Alas}

— São Paulo, SP - Fevereiro de 2002 - 


\section{ESPAÇOS EXPONENCIALMENTE COMPLETOS}

Este exemplar corresponde a versão final da dissertação apresentada por Renato Leme Martin, devidamente corrigida e aprovada pela Comissão Julgadora.

São Paulo, Junho de 2002

\section{Comissão Julgadora}

- Prof $\underline{a}$ Dr $\underline{a}$ Ofélia Teresa Alas (Presidente) - IME-USP

- Prof $\underline{a}$ Dra Lúcia Renato Junqueira - IME-USP

- Prof. Dr. Janey Daccach - UPM 


\section{Abstract}

- The main purpose of this dissertation is to present the exponential space of a topological space (also called hyperspace) and a way to get homeomorphisms in the class of compact metric zero-dimensional spaces. Also it determines the spaces homeomorphic to their own exponential space (called exponentially complete spaces). The theory of accumulation spectra is used to classify the spaces and, with some extra hypotheses to ensure the existence of homeomorphisms. Sierpinski's theorem and the fact that the Cantor space is exponentially complete are obtained as corollaries.

\section{Resumo}

O objetivo principal desta dissertação é apresentar o espaço exponencial de um espaço topológico (também denominado hiperespaço) e um meio de se obter homeomorfismos na classe dos espaços métricos compactos zerodimensionais a fim de determinar os espaços homeomorfos ao seu exponencial, os chamados espaços exponencialmente completos. Para tanto é exibida a teoria dos espectros de acumulação para que os espaços sejam classificados e, mediante algumas hipóteses, garantir homeomorfismos. O teorema de Sierpinski e o fato que o espaço de Cantor é exponencialmente completo aparecem como corolários. 


\section{Sumário}

Introdução

1 Preliminares $\quad 7$

1.1 Espaços Zero-dimensionais . . . . . . . . . . . 7

1.2 Espaços Metrizáveis . . . . . . . . . . . . . . 10

2 Espaços Exponenciais $\quad 12$

3 Teorema do Homeomorfismo de Vaught 17

4 Ordem e Espectro de Acumulação 23

5 Espectros de Acumulação em Espaços Exponenciais 35

A Exponenciais de outros espaços topológicos 43

A.1 $\{0,1\}^{\aleph_{1}} \ldots \ldots \ldots \ldots . \ldots . \ldots . \ldots 44$

A.2 $\{0,1\}^{\aleph_{2}} \ldots \ldots \ldots \ldots . \ldots . \ldots . \ldots . \ldots 4$

A.3 $[0,1] \ldots \ldots \ldots \ldots . \ldots \ldots$

$\begin{array}{ll}\text { Bibliografia } & 47\end{array}$ 


\section{Introdução}

O foco deste trabalho centra-se nos espaços exponenciais: espaços topológicos definidos a partir de outro espaço topológico, em que os pontos deste novo espaço serão os fechados não vazios do espaço de partida. A topologia induzida será a topologia finita ou topologia de Vietoris na qual, cada aberto da nova topologia será definido através de uma quantidade finita de abertos do espaço inicial, sendo formado pelos pontos (fechados na topologia inicial) que estão contidos na união destes abertos e interceptam cada um deles.

A partir daí surge uma questão: existem espaços homeomorfos ao seu próprio exponencial? O título deste trabalho, Espaços exponencialmente completos, refere-se a este tipo de espaço.

Um trabalho realizado por Marjanović [4] em 1972 determina todos ọs espaços que são exponencialmente completos na classe dos espaços métricos compactos zero-dimensionais.

Para se obter os homeomorfismos tem-se como base a teoria dos espectros e ordens de acumulação, na qual o espaço é particionado em vários subconjuntos. A quebra inicia-se separando-se o conjunto dos pontos isolados; em seguida, o conjunto formado pelos pontos tais que toda vizinhança não possui pontos isolados e, desta forma, inicia-se uma construção recursiva. O espaço será classificado conforme seus pontos se distribuem nestes subconjuntos, definindo-se assim o espectro de acumulação do espaço. O homeomorfismo é garantido se dois espaços possuem o mesmo espectro, a mesma quantidade de pontos isolados e o fecho de cada conjunto não vazio da partição (a 
menos do conjunto de pontos isolados) é homeomorfo ao conjunto de Cantor. Veremos que o próprio conjunto de Cantor é um exemplo de um espaço exponencialmente completo.

Uma teoria análoga foi publicada por Vučemilović [16] em 1974 com respeito a espaços métricos zero-dimensionais enumeráveis. É também desenvolvido, por meio dos espectros, um modo de determinar homeomorfismos. O teorema de Sierpinski, que diz que todo espaço métrico enumerável não vazio zero-dimensional sem pontos isolados é homeomorfo ao conjunto dos racionais, acaba saindo como um corolário. Mais tarde, utilizando estes resultados, Vučemilović em conjunto com Marjanović, publicam um artigo [5] no qual são exibidos dois espaços não homeomorfos que possuem quadrados homeomorfos na classe dos espaços métricos zero-dimensionais enumeráveis.

Para garantir os homeomorfismos na classe dos espaços métricos compactos zero-dimensionais, é utilizada uma teoria desenvolvida por R. Vaught na qual define-se um tipo de relação entre espaços que implicará no homeomorfismo, ou seja, se dois espaços satisfazem a relação, são homeomorfos. Stevo Todorcevic em Topics in Topology [13] obtém os homeomorfismos definindo uma relação deste tipo, no caso, ter o mesmo espectro, a mesma quantidade de pontos isolados e a aderência de cada conjunto não vazio da partição citada anteriormente (a não ser o conjunto de pontos isolados) ser homeomorfo ao conjunto de Cantor.

Finalmente, vamos "calcular" o espectro de um espaço exponencial através do espaço de partida e com isso exibir os espaços métricos compactos zerodimensionais que são exponencialmente completos.

No primeiro capítulo apresentaremos alguns resultados importantes relativos a espaços topológicos zero-dimensionais e a espaços metrizáveis, que como pode-se notar, estão presentes em todo o trabalho. Qualquer outra noção topológica não abordada nas preliminares poderá ser encontrada no livro do Engelking [1]. A principal fonte deste trabalho é o livro do Stevo Todorcevic [13] citado anteriormente. 


\section{Capítulo 1}

\section{Preliminares}

Neste primeiro capítulo exibiremos alguns tipos de espaços topológicos e algumas de suas propriedades importantes que serão utilizadas ao longo do texto. Todos os espaços topológicos tratados serão Hausdorff.

Primeiramente trataremos dos espaços zero-dimensionais que estarão presentes durante todo o trabalho.

\subsection{Espaços Zero-dimensionais}

Definição 1.1. Um espaço topológico $X$ é zero-dimensional se possui uma base composta por abertos-fechados.

Teorema 1.2. Todo espaço zero-dimensional não possui nenhum subespaço conexo com cardinalidade maior que 1.

Demonstração. Seja $A$ um subespaço de $X$ tal que $|A|>1$. Tome $x$ e $y$ pontos distintos de $A$ e $U$ aberto-fechado tal que $x \in U$ e $y \notin U$. Assim podemos particionar $A$ em dois subespaços abertos disjuntos $A \backslash U$ e $A \cap U$ concluindo que $A$ não é conexo. 
Lema 1.3. Se $X$ é espaço zero-dimensional com base enumerável então todo aberto pode ser escrito como união disjunta de uma seqüencia de abertosfechados.

Demonstração. Todo aberto $U$ pode ser escrito como união de abertos-fechados da base. Assim, $U=\bigcup_{i=0}^{\infty} U_{i}=\bigcup_{i=0}^{\infty}\left(U_{i} \backslash \bigcup_{j=0}^{i-1} U_{j}\right)$.

Teorema 1.4 (Redução). Seja X um espaço zero-dimensional com base enumerável. Se $G_{0}, \ldots, G_{n}, \ldots$ é uma seqüência (finita ou infinita) de abertos em $X$, então existe uma seqüência $U_{0}, \ldots, U_{n}, \ldots$ de abertos de $X$ dois a dois disjuntos tais que $U_{i} \subset G_{i}$ para todo $i$ e $\bigcup_{i=0}^{\infty} U_{i}=\bigcup_{i=0}^{\infty} G_{i}$.

Demonstração. De acordo com o lema anterior, podemos escrever $G_{i}$ como união disjunta de abertos-fechados $G_{i}=\bigcup_{j=0}^{\infty} U_{i, j}$, para todo $i$. Seja $\left\{W_{i}: i \in \mathbb{N}\right\}$ outra enumeração de $\left\{U_{i, j}: i, j \in \mathbb{N}\right\}$. Definimos $T_{0}=W_{0}$ e $T_{n}=W_{n} \backslash\left(W_{0} \cup \cdots \cup W_{n-1}\right)$ para $n \geq 1$. Obtemos os abertos-fechados desejados da seguinte forma: $U_{0}$ é a reunião dos $T_{n}$ contidos em $G_{0}$, e para $n \geq 1, U_{n}$ é a reunião $\operatorname{dos} T_{k}$ contidos em $G_{n}$ que ainda não foram escolhidos anteriormente para compor algum $U_{m}$ com $m<n$. Note que os abertosfechados $U_{n}$ satisfazem $\bigcup_{i=0}^{\infty} U_{i}=\bigcup_{i=0}^{\infty} G_{i}$ e $U_{i} \subset G_{i}$ para todo $i$.

Teorema 1.5 (Separação). Se $F_{0}, \ldots, F_{n}, \ldots$ é uma seqüência (finita ou infinita) de fechados em um espaço zero-dimensional com base enumerável tal que $\bigcap_{i=0}^{\infty} F_{i}=\varnothing$, então existe $V_{0}, \ldots, V_{n}, \ldots$ uma seqüência de abertosfechados de $X$ tais que $F_{i} \subset V_{i}$ para todo $i$ e $\bigcap_{i=0}^{\infty} V_{i}=\varnothing$. Em particular, se $A$ e $B$ são fechados disjuntos, existe $V$ aberto-fechado tal que $A \subset V e$ $V \cap B=\varnothing$.

Demonstração. Definimos $G_{i}=X \backslash F_{i}$ e, utilizando o teorema anterior, obtemos a seqüência de abertos dois a dois disjuntos $U_{0}, \ldots U_{n}, \ldots$, fazemos 
$V_{i}=X \backslash U_{i}$ e $\operatorname{assim} \bigcup_{i=0}^{\infty} G_{i}=X \backslash \bigcap_{i=0}^{\infty} F_{i}=X=\bigcup_{i=0}^{\infty} U_{i}$. Com isso $U_{i}$ e $V_{i}$ são abertos-fechados para todo $i$ e, através da última igualdade, temos que $\bigcap_{i=0}^{\infty} V_{i}=\varnothing$. A inclusão $U_{i} \subset G_{i}$ nos fornece $F_{i} \subset V_{i}$ para todo $i$.

O conjunto de Cantor $\mathcal{C}$ é um exemplo de espaço métrico compacto zero-dimensional. Sua construção inicia-se a partir do intervalo da reta real $I_{0}=[0,1]$ dividindo-o em três partes iguais e retirando-se o intervalo aberto central. Desta forma obtemos um novo conjunto formado pela união de dois intervalos fechados $I_{1}=\left[0, \frac{1}{3}\right] \cup\left[\frac{2}{3}, 1\right]$. O passo seguinte consiste novamente em dividir cada um dos intervalos em três partes iguais retirando-se os intervalos abertos centrais. Desta forma obtemos $I_{2}=\left[0, \frac{1}{9}\right] \cup\left[\frac{2}{9}, \frac{3}{9}\right] \cup\left[\frac{6}{9}, \frac{7}{9}\right] \cup\left[\frac{8}{9}, 1\right]$ e assim sucessivamente. $\mathrm{O}$ conjunto de Cantor $\mathcal{C}$ será $\mathcal{C}=\bigcap_{n \in \mathbb{N}} I_{n}$. Para ilustrar melhor observe a figura:

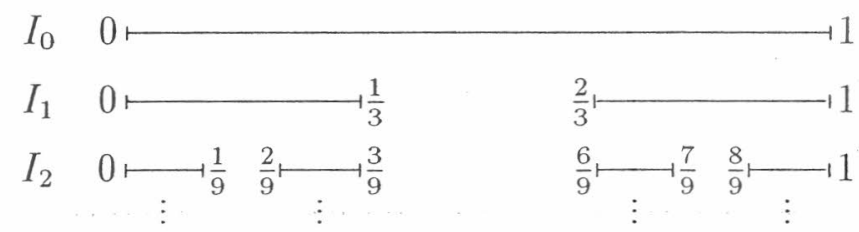

Observe que $\left\{I_{n}: n \in \mathbb{N}\right\}$ é uma família de fechados em $[0,1]$ que satisfaz a propriedade da intersecção finita. $\operatorname{Logo} \mathcal{C}$ é fechado, não vazio e compacto.

Outra característica importante do conjunto de Cantor é a de ser homeomorfo a $\{0,1\}^{\aleph_{0}}$. Isto pode ser verificado observando que cada ponto $x$ de $\mathcal{C}$ pode ser escrito como

$$
x=\sum_{i=0}^{\infty} \frac{2 x_{i}}{3^{i}}, \text { onde } x_{i} \in\{0,1\} \text { e } i \in \mathbb{N}
$$

Com isso definimos $f: \mathcal{C} \mapsto\{0,1\}^{\aleph_{0}}$ como $f(x)=\left(x_{i}\right)_{i \in \mathbb{N}}$ que obviamente trata-se de uma função bijetora e contínua e portanto um homeomorfismo já que $\mathcal{C}$ é compacto. Assim verificamos também que $\mathcal{C}$ é zero-dimensional (teorema 1.8). 
Definição 1.6. Para uma família $\left\{X_{s}: s \in S\right\}$ de espaços topológicos dois a dois disjuntos consideramos o conjunto $X=\bigcup_{x \in S} X_{s}$ e a família $\tau$ de todos os conjuntos $U \subset X$ tais que $U \cap X_{s}$ é aberto em $X_{s}$ para todo $s \in S$. Desta forma $\tau$ define uma topologia em $X$. Ao conjunto $\mathrm{X}$ munido com a topologia $\tau$ damos o nome de soma topológica de $\left\{X_{s}: s \in S\right\}$ e denotamos $\bigoplus_{s \in S} X_{S}$ ou $X_{1} \oplus \cdots \oplus X_{k}$ se $S=\{1, \ldots, k\}$.

Podemos estender a definição de soma topológica para uma família $\left\{X_{s}: s \in S\right\}$ de espaços não disjuntos fazendo $\bigoplus_{s \in S} X_{S}=\bigoplus_{s \in S}\left(X_{S} \times\{s\}\right)$.

Teorema 1.7. A soma $\bigoplus_{s \in S} X_{s}$, onde $S \neq \varnothing$ e $X_{s} \neq \varnothing$ para todo $s \in S$ é zero-dimensional se e só se cada $X_{s}$ é zero-dimensional.

Teorema 1.8. O produto cartesiano $\prod_{s \in S} X_{s}$, onde $S \neq \varnothing$ e $X_{s} \neq \varnothing$ para todo $s \in S$ é zero-dimensional se e só se cada $X_{s}$ é zero-dimensional

A seguir abordaremos os espaços metrizáveis, utilizados no momento em que desejamos inserir uma métrica em um espaço topológico definido a partir da topologia de outro espaço métrico.

\subsection{Espaços Metrizáveis}

Definição 1.9. Um espaço topológico é metrizável se existe uma métrica $d$ em $X$ que gera a mesma topologia de $X$.

Teorema 1.10. Um espaço regular Lindelöf é metrizável se e somente se possui uma base de abertos enumerável. 
Definição 1.11. Seja $(X, d)$ um espaço métrico e $A \subset X$ não vazio. $O$ diâmetro de $\mathbf{A}$ denotado por $\delta(A)$ é o supremo das distâncias dos pontos de $A$, ou seja, $\delta(A)=\sup \{d(x, y): x, y \in A\}$. O diâmetro pode ser $\infty \mathrm{e}$ podemos definir $\delta(\varnothing)=0$.

O teorema a seguir é muito poderoso quando tratamos de espaços métricos completos.

Teorema 1.12 (Intersecção de Cantor). Seja $(X, d)$ um espaço métrico completo. Então para toda seqüência decrescente $F_{0} \supset \cdots \supset F_{n} \supset \ldots$ de fechados não-vazios de $X$ com $\lim _{n \rightarrow \infty} \delta\left(F_{n}\right)=0$, a interseç̧ão $\bigcap_{n=0}^{\infty} F_{n}=\{x\}$ para algum $x \in X$.

Demonstração. A hipótese $\delta\left(F_{n}\right) \rightarrow 0$ garante que a intersecção não pode conter mais de um ponto. Basta mostrarmos que a intersecção não é vazia. Escolhemos $x_{n}$ pertencente a $F_{n}$ para cada $n \in \mathbb{N}$. Como $\delta\left(F_{n}\right) \rightarrow 0$ obtemos $\left(x_{n}\right)_{n \in \mathbb{N}}$ uma seqüência de Cauchy que, por $X$ ser completo, converge para um ponto $x \in X$.

Vamos mostrar que $x$ pertence a $F_{n}$ para todo $n \in \mathbb{N}$. Seja $n_{0} \in \mathbb{N}$ arbitrário. Como $F_{0} \supset \cdots \supset F_{n} \supset \ldots$, os pontos da seqüência $\left(x_{n}\right)_{n \geq n_{0}}$ pertencem todos a $F_{n_{0}}$. Como $F_{n_{0}}$ é fechado, $x$, que é limite desta seqüencia, pertence a $F_{n_{0}}$.

Obs 1.13. O teorema anterior será empregado nesta dissertação em espaços métricos compactos, já que todo espaço métrico compacto é completo 


\section{Capítulo 2}

\section{Espaços Exponenciais}

Primeiramente iremos definir espaço exponencial de um espaço topológico, apresentar suas principais propriedades e alguns resultados.

Definição 2.1. O espaço exponencial $\exp (X)$ (também chamado de hiperespaço e denotado por $2^{X}$ ) de um espaço topológico $X$ é formado pelo conjunto de todos os fechados não vazios de $X$ munido com a topologia gerada por dois tipos de abertos sub-básicos:

$$
\begin{aligned}
\langle U\rangle & =\{F \in \exp (X) \mid F \subseteq U\} \\
\rangle U\langle & =\{F \in \exp (X) \mid F \cap U \neq \varnothing\}
\end{aligned}
$$

onde $U$ é um aberto não vazio de $X$.

Temos, assim, uma base para a topologia formada por elementos do tipo

$$
\left\langle U_{1}, \ldots, U_{n}\right\rangle=\left\{F \in \exp (X) \mid F \subseteq \bigcup_{i=1}^{n} U_{i} \text { e } F \cap U_{i} \neq \varnothing, 1 \leq i \leq n\right\}
$$

onde $U_{1}, \ldots, U_{n}$ são abertos não vazios de $X$.

Note que

$$
\left.\left\langle U_{1}, \ldots, U_{n}\right\rangle=\left\langle\bigcup_{i=1}^{n} U_{i}\right\rangle \cap \bigcap_{i=1}^{n}\right\rangle U_{i}\langle
$$


Esta topologia é chamada de topologia de Vietoris ou topologia finita.

Podemos também definir uma sub-base de fechados da seguinte forma:

$$
\begin{aligned}
& \langle F\rangle=\exp (X) \backslash\rangle X \backslash F \backslash=\left\{F^{\prime} \in \exp (X) \mid F^{\prime} \subset F\right\} \\
& \rangle F\left\langle=\exp (X) \backslash\langle X \backslash F\rangle=\left\{F^{\prime} \in \exp (X) \mid F^{\prime} \cap F \neq \varnothing\right\}\right.
\end{aligned}
$$

para $F$ fechado não vazio em $X$.

Podemos ressaltar alguns subespaços de $\exp (X)$ importantes como $[X] \leq n$ formado pelos conjuntos finitos de $X$ com no máximo $n$ elementos. São chamados de enésima potência simétrica de $X$. Observe que $[X]^{\leq 1}$ é um subespaço de $\exp (X)$ homeomorfo a $X$ (através do homeomorfismo natural $x \mapsto\{x\})$ e que $\bigcup_{n \in \mathbb{N}}[X] \leq n$ é denso em $\exp (X)$.

A seguir, exibiremos algumas propriedades preservadas pelo exponencial. Proposição 2.2. $X$ é compacto se e somente se $\exp (X)$ é compacto.

Demonstração. $(\Rightarrow)$ Utilizando o teorema da sub-base de Alexander [1] vamos mostrar que uma família $\mathcal{F}$ de elementos não-vazios de uma sub-base fechada de $\exp (X)$ com a propriedade da intersecção finita (p.i.f.) possui intersecção não vazia. $\mathcal{F}$ pode ser particionado em $\mathcal{F}_{0}$ e $\mathcal{F}_{1}$, onde $\mathcal{F}_{0}$ é formado por elementos do tipo $\langle F\rangle$ e $\mathcal{F}_{1}$ por elementos da forma $\rangle F\langle$. Observando que $\left\{F:\langle F\rangle \in \mathcal{F}_{0}\right\}$ satisfaz p.i.f. (dado que $\mathcal{F}_{0}$ satisfaz) e a compacidade de $\mathrm{X}$ obtemos

$$
\varnothing \neq F_{0}=\bigcap\left\{F:\langle F\rangle \in \mathcal{F}_{0}\right\}
$$

Precisamos mostrar que $F_{0} \in \cap \mathcal{F}_{1}$.

Se $\left.F_{0} \notin\right\rangle F^{\prime}\langle$, para algum $\rangle F^{\prime}\left\langle\in \mathcal{F}_{1}\right.$, temos que $F_{0} \cap F^{\prime}=\varnothing$. Pela. compacidade de $X$, existem $F_{1}, \ldots, F_{p} \in\left\{F:\langle F\rangle \in \mathcal{F}_{0}\right\}$ que satisfazem $F_{1} \cap \cdots \cap F_{p} \cap F^{\prime}=\varnothing$. Com isso, teríamos $\left.\left\langle F_{1}\right\rangle \cap \cdots \cap\left\langle F_{p}\right\rangle \cap\right\rangle F^{\prime}\langle=\varnothing$, o que é um absurdo já que $\mathcal{F}$ satisfaz p.i.f.

$(\Leftarrow)$ Supondo $\exp (X)$ compacto e seja $\left\{U_{s}: s \in S\right\}$ uma cobertura aberta de $X$. Temos que $\left\{\left\langle X, U_{s}\right\rangle: s \in S\right\}$ é uma cobertura aberta de $\exp (X)$ que 
admite $\left\{\left\langle X, U_{1}\right\rangle, \ldots,\left\langle X, U_{p}\right\rangle\right\}$ subcobertura finita. Obtemos $\left\{U_{1}, \ldots, U_{p}\right\}$ subcobertura finita de $X$ concluindo que $X$ é compacto.

Proposição 2.3. X é compacto métrico se e somente se $\exp (X)$ é compacto métrico

Demonstração. $(\Rightarrow)$ Sendo $\beta$ uma base enumerável de $X$, podemos construir $\left\{\left\langle U_{1}, \ldots, U_{n}\right\rangle: n \in \mathbb{N}, U_{i} \in \beta\right.$ para $\left.1 \leq i \leq n\right\}$ uma base enumerável de $\exp (X)$ já que $X$ é compacto. Logo $\exp (X)$ é metrizável.

$(\Leftarrow)$ Sendo $\exp (X)$ métrico, o mesmo acontece com seu subespaço $[X] \leq 1$ que é homeomorfo a $X$ sendo portanto também métrico. Dada a compacidade de $\exp (X)$ obtemos a compacidade de $X$.

Proposição 2.4. X é zero-dimensional se e só se $\exp (X)$ é zero-dimensional.

Demonstração. $(\Rightarrow)$ Basta observar que se $U$ é um aberto-fechado de $X$ então $\langle U\rangle$ e $\rangle U\langle$ são abertos-fechados $\operatorname{em~} \exp (X)$.

$(\Leftarrow)$ Imediato ao observarmos o subespaço $[X]^{1}$ de $\exp (X)$ que é homeomorfo a $X$.

Proposição 2.5. Sejam $X$ compacto, $g: X \rightarrow Y$ uma função contínua. Então $F \mapsto g[F]$ é uma função continua de $\exp (X)$ em $\exp (Y)$.

Demonstração. Definimos $G: \exp (X) \rightarrow \exp (Y)$ como $G(F)=g[F]$. $G$ está bem definida pois como $F$ é fechado em $X$ que é compacto, $F$ é compacto. Pela continuidade de $g$ temos que $g[F]$ é compacto em $Y$ e portanto fechado (os espaços são Hausdorff), ou seja, $g[F] \in \exp (Y)$. 
Seja $F_{Y} \subseteq Y$ fechado e $F_{X}=g^{-1}\left[F_{Y}\right]$, mostraremos que $G^{-1}\left[\left\langle F_{Y}\right\rangle\right]=\left\langle F_{X}\right\rangle$. Cada coluna abaixo representa um lado da inclusão e cada linha é conseqüência da anterior.

$$
\begin{array}{c|c}
F \in G^{-1}\left[\left\langle F_{Y}\right\rangle\right] & F \in\left\langle F_{X}\right\rangle \\
G(F) \in\left\langle F_{Y}\right\rangle & F \in\left\langle g^{-1}\left[F_{Y}\right]\right\rangle \\
g[F] \in\left\langle F_{Y}\right\rangle & F \subset g^{-1}\left[F_{Y}\right] \\
g[F] \subset F_{Y} & g[F] \subset g\left[g^{-1}\left[F_{Y}\right]\right] \subset F_{Y} \\
F \subset g^{-1}[g[F]] \subset g^{-1}\left[F_{Y}\right] & g[F] \in\left\langle F_{Y}\right\rangle \\
F \in\left\langle F_{X}\right\rangle & F \in G^{-1}\left[\left\langle F_{Y}\right\rangle\right] \\
\therefore G^{-1}\left[\left\langle F_{Y}\right\rangle\right] \subset\left\langle F_{X}\right\rangle & \therefore\left\langle F_{X}\right\rangle \subset G^{-1}\left[\left\langle F_{Y}\right\rangle\right]
\end{array}
$$

Da mesma maneira, vamos mostrar que $\left.G^{-1}[\rangle F_{Y}\langle]=\right\rangle F_{X}\langle$.

$$
\begin{array}{c|c}
F \in G^{-1}[\rangle F_{Y}\langle] & F \in\rangle F_{X}\langle \\
g[F] \in\rangle F_{Y}\langle & F \cap F_{X} \neq \varnothing \\
y \in g[F] \cap F_{Y} \neq \varnothing & F \cap g^{-1}\left[F_{Y}\right] \neq \varnothing \\
\exists x \in F \mid g(x)=y & g[F] \cap g\left[g^{-1}\left[F_{Y}\right]\right] \neq \varnothing \\
x \in F \cap g^{-1}\left[F_{Y}\right] & g[F] \cap F_{Y} \neq \varnothing \\
F \in\rangle g^{-1}\left[F_{Y}\right]< & g[F] \in\rangle F_{Y}\langle \\
F \in\rangle F_{X}\langle & G(F) \in\rangle F_{Y}\langle \\
\left.G^{-1}[\rangle F_{Y}\langle] \subset\right\rangle F_{X}\langle & F \in G^{-1}[\rangle F_{Y}\langle] \\
& >F_{X}\left\langle\subset G^{-1}[\rangle F_{Y}\langle]\right.
\end{array}
$$

Podemos também determinar os pontos isolados e de acumulação do espaço exponencial de $X$ com base nos pontos isolados e de acumulação de $X$. A seguir temos duas proposições a esse respeito. 
Proposição 2.6. Seja $x$ ponto de acumulação de $X$ e $F \in \exp (X)$. Se $x \in F$ então $F$ é ponto de acumulação de $\exp (X)$.

Demonstração. Supondo que $F$ seja isolado em $\exp (X)$, devem existir $U_{1}, \ldots, U_{n}$ abertos de $X$ que satisfaçam

$$
\{F\}=\left\langle U_{1}, \ldots, U_{n}\right\rangle
$$

Logo $x \in U_{k}$ para algum $k, 1 \leq k \leq n$. Como $x$ é ponto de acumulação de $X, U_{k}$ é infinito. Se $F$ é finito, escolhemos $x_{k} \in U_{k} \backslash F$, caso contrário, escolhemos $x_{k} \in U_{k}$ qualquer. Para cada $i \neq k$ escolhemos $x_{i} \in U_{i}$. Assim, obtemos $\left\{x_{1}, \ldots, x_{n}\right\} \in\left\langle U_{1}, \ldots, U_{n}\right\rangle$ diferente de $F$ contrariando a hipótese de que $F$ é isolado. $\operatorname{Logo} F$ é ponto de acumulação de $\exp (X)$.

Proposição 2.7. Se $F$ é finito formado apenas por pontos isolados de $X$ então $F$ é ponto isolado de $\exp (X)$.

Demonstração. Seja $F=\left\{x_{1}, \ldots, x_{n}\right\}$. Verificamos a afirmação se observarmos que $\{F\}=\left\langle\left\{x_{1}\right\}, \ldots,\left\{x_{n}\right\}\right\rangle$.

Obs 2.8. Se $X$ é compacto todo ponto isolado de $\exp (X)$ é um conjunto finito de pontos isolados de $X$.

Definição 2.9. Um espaço topológico $X$ é exponencialmente completo se é homeomorfo a $\exp (X)$.

Veremos mais adiante que o conjunto de Cantor é exponencialmente completo. Já o espaço dos racionais $\mathbb{Q}$ não é, pois se observarmos $\mathbb{N} \subset \mathbb{Q}$, que é um subespaço fechado discreto, podemos verificar que

$$
|\exp (\mathbb{Q})| \geq|\exp (\mathbb{N})|=2^{\aleph_{0}}>|\mathbb{Q}|
$$




\section{Capítulo 3}

\section{Teorema do Homeomorfismo de Vaught}

Este teorema visa caracterizar espaços homeomorfos na classe dos espaços métricos compactos zero-dimensionais $\left(\mathcal{Z}_{0}\right)$.

Definiremos um tipo de relação que garantirá o homeomorfismo entre dois espaços, ou seja, se eles satisfazem a relação, são homeomorfos.

Definição 3.1. Uma relação binária $\mathrm{R}$ em $\mathcal{Z}_{0}$ é uma relação de Vaught se satisfaz as seguintes condições:

a) $X R Y$ implica $Y R X$

b) $X R \varnothing$ implica $X=\varnothing$

c) se $X R\left(Y_{0} \oplus Y_{1}\right)$ então existe uma decomposição $X=X_{0} \oplus X_{1}$ tal que $X_{0} R Y_{0}$ e $X_{1} R Y_{1}$

Teorema 3.2 (Vaught). Sejam $X, Y \in \mathcal{Z}_{0}$ tal que $X R Y$ para alguma relação de Vaught $R$ em $\mathcal{Z}_{0}$. Então $X$ é homeomorfo a $Y$.

Demonstração. Vamos pensar em $X$ e $Y$ como subespaços de $\{0,1\}^{\aleph_{0}}$ e fazer uma construção recursiva para gerarmos abertos-fechados $X_{\sigma}$ em $X$ e $Y_{\sigma}$ em $Y$, com $\sigma$ em $\{0,1\}^{<\infty}$, tais que $X_{\sigma} R Y_{\sigma}$. 
Primeiramente fazemos $X_{\varnothing}=X$ e $Y_{\varnothing}=Y$. Suponha que $X_{\sigma}$ e $Y_{\sigma}$ estão definidos com $X_{\sigma} R Y_{\sigma}$. Vamos dividir a construção em dois casos:

- $|\sigma|$ é par

Vamos construir $X_{\sigma 0} \oplus X_{\sigma 1}=X_{\sigma}$ com $X_{\sigma 0}$ e $X_{\sigma 1}$ abertos-fechados tais que $\operatorname{diam}\left(X_{\sigma 0}\right), \operatorname{diam}\left(X_{\sigma 1}\right)<\frac{2}{3} \operatorname{diam}\left(X_{\sigma}\right)$. Utilizaremos em $\{0,1\}^{\mathbb{N}}$ a métrica:

$$
d(x, z)=2^{-\min \{n: x(n) \neq z(n)\}}
$$

Seja $t$ a seqüência maximal em $\{0,1\}^{<\infty}$ tal que $X_{\sigma} \subset[t]$. Se $m$ é o comprimento de $t$ então $2^{-m-1}$ é o diâmetro de $X_{\sigma}$ porque min $\{n: x(n) \neq z(n)\} \geq$ $m+1$ para todo $x, z \in X_{\sigma}$. Sejam

$$
X_{\sigma 0}=X_{\sigma} \cap[t 0] \text { e } X_{\sigma 1}=X_{\sigma} \cap[t 1]
$$

Como t é maximal, geramos conjuntos não vazios e para $i=0,1$ e $x, z \in X_{\sigma i}$ temos $\min \{n: x(n) \neq z(n)\} \geq m+2$.

$\operatorname{Assim} \operatorname{diam}\left(X_{\sigma 0}\right), \operatorname{diam}\left(X_{\sigma 1}\right) \leq 2^{-m-2}<\frac{2}{3} \operatorname{diam}\left(X_{\sigma}\right)$.

Como $X_{\sigma} R Y_{\sigma}$, existe uma decomposição $Y_{\sigma}=Y_{\sigma 0} \oplus Y_{\sigma 1}$ tal que $X_{\sigma 0} R Y_{\sigma 0}$ e $X_{\sigma 1} R Y_{\sigma 1}$.

- $|\sigma|$ é ímpar

Repetimos o procedimento anterior invertendo os papéis de $X$ e $Y$ para garantirmos não só a diminuição do diâmetro dos $X_{\sigma}$ 's, mas também do diâmetro do $Y_{\sigma}$ 's.

Desta forma construímos uma árvore de raiz $X$ e uma árvore com raiz $Y$, onde a cada aberto-fechado $X_{\sigma}$ da primeira árvore associa-se um abertofechado $Y_{\sigma}$ correspondente da segunda árvore com $X_{\sigma} R Y_{\sigma}$. 
Veja a figura:
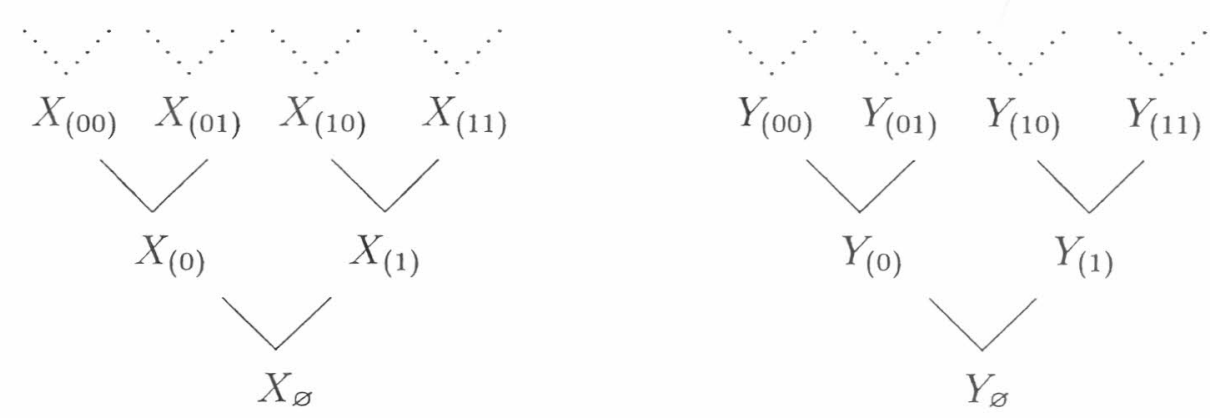

Vamos definir um homeomorfismo $\phi: X \rightarrow Y$. Para cada $x \in X$ existe um único ramo infinito $\sigma_{0} \subset \sigma_{1} \subset \sigma_{2} \subset \ldots$ de $\{0,1\}<\infty$ tal que $x \in X_{\sigma_{n}}$ para todo $n \in \mathbb{N}$. Como $Y$ é compacto, cada $Y_{\sigma_{n}}$ é fechado com $Y_{\sigma_{n}} \supset Y_{\sigma_{n+1}}$ e $\operatorname{diam}\left(Y_{\sigma_{n}}\right) \rightarrow 0$, temos um único $y$ tal que:

$$
\{y\}=\bigcap_{n=0}^{\infty} Y_{\sigma_{n}}
$$

Definimos:

$$
\phi(x)=y
$$

Vamos mostrar que $\phi$ é um homeomorfismo. Pela compacidade de $X$ e $Y$ basta verificarmos que $\phi$ é bijetora e contínua.

- $\phi$ é injetora: Diferentes ramos na árvore com raiz $Y$ correspondem a diferentes pontos de $Y$.

- $\phi$ é sobrejetora: Todo ponto de $Y$ corresponde a um ramo da árvore construída.

- $\phi$ é contínua: Seja $U$ um aberto-fechado tal que $y \in U$ e $\phi(x)=y$. Dado que $\operatorname{diam}\left(Y_{\sigma_{n}}\right) \rightarrow 0$ existe $k$ tal que $Y_{\sigma_{k}} \subseteq U$. Logo $\phi\left[X_{\sigma_{k}}\right] \subseteq$ $Y_{\sigma_{k}} \subseteq U$; além disso $x \in X_{\sigma_{k}}$.

Portanto $\phi$ é um homeomorfismo de $X$ em $Y$. 
Vejamos agora algumas aplicações deste teorema ao definirmos relações de Vaught obtendo classes de espaços homeomorfos.

Corolário 3.3 (G. Cantor, L. E. J. Brouwer). O conjunto de Cantor é o único espaço (não vazio) métrico compacto zero-dimensional sem pontos isolados.

Demonstração. Basta definirmos a seguinte relação de Vaught $R: X R \varnothing$ se $X=\varnothing$; para $X$ e $Y$ não vazios, $X R Y$ se e só se $X$ e $Y$ não possuem pontos isolados. Se $X=X_{0} \oplus X_{!}$, dividimos $Y$ em abertos-fechados $Y_{0}$ e $Y_{1}$ tais que $Y=Y_{0} \oplus Y_{1}$ e para $i=0,1$ pomos $Y_{i}=\varnothing$ se e só se $X_{i}=\varnothing$. Assim temos $X_{0} R Y_{0}$ e $X_{1} R Y_{1}$.

Desta forma $R$ define uma relação de Vaught e com isso o conjunto de Cantor é o "único" espaço em $\mathcal{Z}_{0}$ sem pontos isolados.

Com base na construção do conjunto de Cantor podemos criar outro espaço que contém um conjunto denso de pontos isolados e o restante homeomorfo ao conjunto de Cantor. Basta ao retirarmos cada intervalo, mantermos seu ponto médio. Este espaço é conhecido como espaço de Pelczynski e denotado por $\mathcal{C}_{2}$.

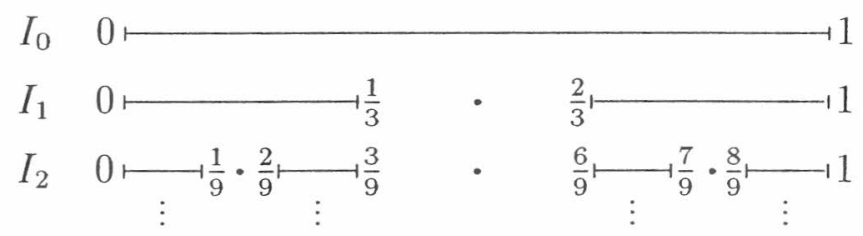

Corolário 3.4 (Pelczynski). O espaço de Pelczynski é o único espaço compacto métrico zero-dimensional com um conjunto denso de pontos isolados e o restante homeomorfo ao conjunto de Cantor.

Demonstração. Novamente vamos definir uma relação de Vaught apropriada para caracterizar os espaços deste tipo. Definimos $X R \varnothing$ se $X=\varnothing$. Para $X$ e $Y$ não-vazios definimos $X R Y$ se ambos possuem um conjunto denso de 
pontos isolados e o restante homeomorfo ao conjunto de Cantor ou se $X$ e $Y$ são finitos com o mesmo número de elementos.

Para verificarmos a condição c. suponha $X R Y$ e que $X$ pode ser decomposto em dois conjuntos abertos-fechados $X_{0}$ e $X_{1}$. Se $X$ é vazio ou finito, $Y$ também deve ser. Supondo que $X$ e $Y$ possuam conjunto denso de pontos isolados e o resto homeomorfo ao conjunto de Cantor. Se $X_{0}$ e $X_{1}$ interceptam a cópia do conjunto de Cantor dentro de $X$, então divida $Y$ em dois abertos-fechados $Y_{0}$ e $Y_{1}$ ambos interceptando a cópia do conjunto de Cantor em $Y$. Assim, $X_{i} R Y_{i}$ para $i=0,1$. Se $X_{0}$ não intercepta o conjunto de Cantor, é formado apenas com pontos isolados e deve ser finito dado que é compacto. Neste caso, escolha o conjunto $Y_{0}$ com o mesmo número de elementos de $X$ também formado apenas de pontos isolados. Faça $Y_{1}=Y \backslash Y_{0}$ e assim temos $X_{i} R Y_{i}$ para $i=0,1$. Logo $R$ é uma relação de Vaught em $\mathcal{Z}_{0}$ e conseqüentemente o espaço de Pelczynski é o "único" elemento de $\mathcal{Z}_{0}$ que satisfaz as condições do enunciado.

A seguir apresentamos alguns exemplos de aplicação dos corolários anteriores.

Exemplo 3.5. $\left.\exp \left(\{0\} \cup\left\{\frac{1}{n}: n \in \mathbb{N}^{*}\right\}\right)\right)$ é homeomorfo a $C_{2}$.

Demonstração. Vamos utilizar o corolário anterior (Pelczynski) para mostrar este fato. Para $X=\{0\} \cup\left\{\frac{1}{n}: n \in \mathbb{N}^{*}\right\}$, mostraremos que o conjunto dos pontos isolados em $\exp (X)$ é denso e que o restante é homeomorfo ao conjunto de Cantor.

Destacamos que $X$ é compacto e conseqüentemente $\exp (X)$ também e que os pontos isolados de $\exp (X)$ são conjuntos finitos contendo apenas pontos isolados de $X$, ou seja, qualquer ponto de $X$ diferente de 0 .

Em qualquer $\left\langle U_{1}, \ldots, U_{n}\right\rangle$ aberto $\operatorname{de} \exp (X)$ podemos escolher $x_{i} \in U_{i} \backslash\{0\}$, para cada $i$, obtendo $\left\{x_{1}, \ldots, x_{n}\right\}$ ponto isolado de $\exp (X)$ pertencente a $\left\langle U_{1}, \ldots, U_{n}\right\rangle$. Logo o conjunto de pontos isolados é denso $\exp (X)$. 
Vamos analisar o subespaço $Y=\exp (X) \backslash\{F \in \exp (X): 0 \notin F$ e $F$ é finito $\}$. Como tiramos todos os pontos isolados, o restante é fechado em $\exp (X)$ e portanto compacto.

Para $F \in Y$, temos que $0 \in F$. Se $F$ fosse isolado em $Y$, existiriam $U_{1}, \ldots, U_{n}$ abertos em $X$ tais que $\{F\}=\left\langle U_{1}, \ldots, U_{n}\right\rangle \cap Y$ e assim, para algum $k, 1 \leq k \leq n$ teríamos $0 \in U_{k}$, um aberto infinito. Sendo $F$ finito, escolhemos $x_{k} \in U_{k} \backslash F$ e caso contrário $x_{k} \in U_{k}$ qualquer. Para $i \neq k$, escolhemos $x_{i} \in U_{i}$. Construímos o conjunto $\{0\} \cup\left\{x_{1}, \ldots, x_{n}\right\}$ que pertence a $\left\langle U_{1}, \ldots, U_{n}\right\rangle \cap Y$ e é diferente de $F$. Logo $F$ não é isolado em $Y$.

Retirando os pontos isolados de $\exp (X)$, restam apenas pontos não isolados em $Y$ que é compacto e, de acordo com outro corolário (Cantor), é homeomorfo ao conjunto de Cantor. Logo $\exp (X)$ é homeomorfo ao espaço de Pelczynski $\left(C_{2}\right)$.

Exemplo 3.6. $\exp \left(C_{2}\right)$ é homeomorfo a $C_{2}$.

Demonstração. Primeiramente dividimos $C_{2}=K \cup A$, onde $K$ é homeomorfo ao conjunto de Cantor e $A$ é o conjunto de pontos isolados de $C_{2}$.

Observe que os pontos isolados de $\exp \left(C_{2}\right)$ são subconjuntos finitos $\operatorname{de} A$ e que o conjunto dos pontos isolados de $\exp \left(C_{2}\right)$ é denso já que todo aberto de $C_{2}$ possui ponto isolado (para $\left\langle U_{1}, \ldots, U_{n}\right\rangle$ aberto $\operatorname{em} \exp \left(C_{2}\right)$ escolhemos um ponto isolado de cada $U_{i}$ formando um conjunto finito de pontos isolados de $C_{2}$ e portanto isolado em $\left.\exp \left(C_{2}\right)\right)$.

Seja $Y=\exp \left(C_{2}\right) \backslash\left\{F \in \exp \left(C_{2}\right): F\right.$ é isolado em $\left.\exp \left(C_{2}\right)\right\}$. Se $F \in Y$, $F \cap K \neq \varnothing$. Seja $x \in F \cap K$. Se $F$ fosse isolado em $Y$, existiriam $U_{1}, \ldots, U_{n}$ abertos em $C_{2}$, tais que $\{F\}=\left\langle U_{1}, \ldots, U_{n}\right\rangle \cap Y$. Logo, para algum $k$ teríamos, $x \in U_{k}$ infinito. Se $F$ é finito, escolhemos $x_{k} \in U_{k} \backslash F$ e se $F$ é infinito, $x_{k} \in U_{k}$ qualquer. Desta forma $F \neq\{x\} \cup\left\{x_{1}, \ldots, x_{n}\right\} \in\left\langle U_{1}, \ldots, U_{n}\right\rangle \cap Y$ e assim $F$ não pode ser isolado em $Y$. Retirando os pontos isolado de $C_{2}$ não resta nenhum ponto isolado no subespaço que é compacto e portanto homeomorfo ao conjunto de Cantor. Desta forma, concluímos que $\exp \left(C_{2}\right)$ é homeomorfo a $C_{2}$. 


\section{Capítulo 4}

\section{Ordem e Espectro de Acumulação}

A seguir será apresentada a teoria dos espectros e ordens de acumulação de espaços topológicos a fim de estabelecermos caracterizações de homeomorfismos entre espaços métricos zero-dimensionais.

Iremos particionar o espaço separando inicialmente os pontos isolados e, a partir daí, definir recursivamente os demais conjuntos. Definiremos a ordem de acumulação de um ponto do espaço conforme sua localização, e caracterizaremos o espaço através de seu espectro de acumulação, que corresponderá a ordem dos pontos deste conjunto. Com base nestes aspectos, definiremos uma relação de Vaught para determinarmos os homeomorfismos.

A seguir exibimos o processo de construção. 
Seja $X$ um espaço topológico,

$$
\begin{aligned}
X^{(0)}= & \text { conjunto dos pontos isolados de } X \\
X^{(1)}= & X \backslash \overline{X^{(0)}} \\
\vdots & \vdots \\
X^{(n)}= & \left(\overline{X^{(n-2)}} \backslash X^{(n-2)}\right) \backslash \overline{X^{(n-1)}} \text {, para } n \geq 2 \\
X^{(\infty)}= & \bigcap_{n=0}^{\infty} \overline{X^{(n)}}
\end{aligned}
$$

Exemplos.

1. Para $X=\{0\} \cup\{1 / n: n \in \mathbb{N}\}$, temos $X^{(0)}=\{1 / n: n \in \mathbb{N}\}, X^{(1)}=\varnothing$ e $X^{(2)}=\{0\}$

2. Se $X$ é espaço de Cantor, então $X^{(0)}=\varnothing$ e $X^{(1)}=\mathcal{C}$

3. Se $X$ é o espaço de Pelczynski $\left(\mathcal{C}_{2}\right)$, então $X^{(0)}$ é o conjunto dos pontos médios dos intervalos removidos, $X^{(1)}=\varnothing \mathrm{e} X^{(2)}=\mathcal{C}$

\section{Obs 4.1.}

1. Como $X^{(0)}$ é o conjunto dos pontos isolados, e, se um ponto isolado pertence à aderência de um conjunto, pertence também ao conjunto, pelo processo de construção anterior, temos que $X^{(0)}$ é disjunto de $X^{(n)}$ para todo $n \geq 1$.

2. $X^{(1)}$ é o subespaço de todos os pontos que possuem uma vizinhança sem pontos isolados.

3. Observando a definição, verificamos as seguintes inclusões

$$
\begin{aligned}
& \overline{X^{(1)}} \supset \overline{X^{(3)}} \supset \cdots \supset \overline{X^{(2 k+1)}} \supset \ldots \\
& \overline{X^{(2)}} \supset \overline{X^{(4)}} \supset \cdots \supset \overline{X^{(2 k)}} \supset \ldots
\end{aligned}
$$


Inicialmente, veremos que foi definida uma cobertura de $X$, ou seja, que $X=\bigcup_{0 \leq n \leq \infty} X^{(n)}$. Supondo que $x \in X$ e $x \notin X^{(\infty)}=\bigcap_{n \geq 0} \overline{X^{(n)}}$, existe $m$ mínimo tal que $x \notin \overline{X^{(m)}}$.

Se $m=0, x \in X \backslash \overline{X^{(0)}}=X^{(1)}$.

Se $m>0$, temos $x \notin \overline{X^{(m)}}$ e $x \in \overline{X^{(m-1)}}$. Concluímos que $x \in X^{(m-1)}$ ou $x \in X^{(m+1)}$ ao observarmos a definição $X^{(m+1)}=\left(\overline{X^{(m-1)}} \backslash X^{(m-1)}\right) \backslash \overline{X^{(m)}}$.

Para verificarmos que esta cobertura é disjunta, vamos demonstrar a proposição seguinte com a qual este fato se torna imediato.

Proposição 4.2. $\complement \overline{X^{(n)}}=X^{(0)} \cup \cdots \cup X^{(n-1)} \cup X^{(n+1)}$ para $1 \leq n<\infty$ Demonstração. Para $n=1$, temos $\complement \overline{X^{(1)}}=X^{(0)} \cup X^{(2)}$.

$(\subseteq) \overline{X^{(1)}}=\overline{X \backslash \overline{X^{(0)}}}=X \backslash \overline{X^{(0)}}$. Se $x \notin \overline{X^{(1)}}$ então $x \in \overline{X^{(0)}}$, ou seja, $x \in \overline{X^{(0)}}$. Observando $X^{(2)}=\left(\overline{X^{(0)}} \backslash X^{(0)}\right) \backslash \overline{X^{(1)}}$, segue que $x \in X^{(0)}$ ou $x \in X^{(2)}$.

$(\supseteq)$ Como $X^{(0)}$ e $X^{(1)}$ são abertos disjuntos, $X^{(0)}$ não encontra $\overline{X^{(1)}}$, e, pela definição, $X^{(2)}$ não encontra $\overline{X^{(1)}}$.

Para $n \geq 2$

$(\subseteq)$ Se $y \notin \overline{X^{(n)}}$ temos duas possibilidades. Ou $n$ é mínimo para esta propriedade, ou existe $p<n$, mínimo, tal que $y \notin \overline{X^{(p)}}$. Então, pelas definições de $X^{(n)}$ e $X^{(p)}$ temos respectivamente,

$$
\left\{\begin{array} { c } 
{ y \in X ^ { ( n - 1 ) } } \\
{ \text { ou } } \\
{ y \in X ^ { ( n + 1 ) } }
\end{array} \quad \text { ou } \quad \left\{\begin{array}{c}
y \in X^{(p-1)} \\
\text { ou } \\
y \in X^{(p+1)}
\end{array}\right.\right.
$$

$\log 0$

$$
\complement \overline{X^{(n)}} \subseteq X^{(0)} \cup \cdots \cup X^{(n-1)} \cup X^{(n+1)}
$$

() Utilizaremos a indução sobre $n$. Supondo a inclusão para valores menores que $n$ e observando que $\overline{X^{(n)}} \subset \overline{X^{(n-2)}}$ e $\overline{X^{(n)}} \cap X^{(n+1)}=\varnothing$, concluímos 
que $\complement \overline{X^{(n)}} \supseteq X^{(0)} \cup \cdots \cup X^{(n-3)} \cup X^{(n-1)} \cup X^{(n+1)}$. Falta mostrarmos que $\overline{X^{(n)}} \cap X^{(n-2)}=\varnothing$.

Pela definição, $X^{(n)} \cap X^{(n-2)}=\varnothing$ e, através da hipótese de indução, obtemos $\complement \overline{X^{(n-3)}}=X^{(0)} \cup \cdots \cup X^{(n-4)} \cup X^{(n-2)}$, um aberto que contém $X^{(n-2)}$ e não encontra $X^{(n)}$.

Logo $\complement \overline{X^{(n)}} \subseteq X^{(0)} \cup \cdots \cup X^{(m-1)} \cup X^{(m+1)}$ verificando a igualdade.

Corolário 4.3. Seja $X$ um espaço topológico. Então $X^{(i)} \cap X^{(j)}=\varnothing$ para todo $i \neq j$

Demonstração. Basta observar a proposição anterior na qual $\overline{X^{(n)}}$ não encontra $X^{(i)}$ para todo $i<n$. Conseqüentemente, $X^{(\infty)} \cap X^{(i)}=\varnothing$, para todo $i<\infty$.

Corolário 4.4. Seja $X$ um espaço topológico. Então $\overline{X^{(n)}}=X^{(n)} \cup \bigcup_{n+2 \leq k \leq \infty} X^{(k)}$ $\operatorname{para} n \geq 0$.

Demonstração. Este fato é verificado passando ao complementar nos dois lados da expressão da proposição anterior, juntamente com corolário anterior.

Corolário 4.5. Seja $X$ um espaço topológico. Então, para $n \geq 2$, $X^{(n)}=\left(X \backslash \bigcup_{k=0}^{n-2} X^{(k)}\right) \backslash \overline{X^{(n-1)}}$

Demonstração. Basta utilizarmos a definição e o corolário anterior

$$
\begin{aligned}
& X^{(n)}=\left(\overline{X^{(n-2)}} \backslash X^{(n-2)}\right) \backslash \overline{X^{(n-1)}} \\
& X^{(n)}=\left(X^{(n-2)} \cup \bigcup_{n \leq k \leq \infty} X^{(k)} \backslash X^{(n-2)}\right) \backslash \overline{X^{(n-1)}} \\
& X^{(n)}=\bigcup_{n \leq k \leq \infty} X^{(k)} \sqrt{X^{(n-1)}} \\
& X^{(n)}=\left(X \backslash \bigcup_{k=0}^{n-2} X^{(k)}\right) \backslash \overline{X^{(n-1)}}
\end{aligned}
$$


Corolário 4.6. $X^{(n)}=\varnothing$ implica $X^{(k)}=\varnothing$ para todo $k \geq n+2$

Demonstração. Conseqüência imediata do corolário 4.4.

Corolário 4.7. $\bigcup_{k=0}^{n} X^{(k)} e \bigcup_{k=0}^{n-2} X^{(k)} \cup X^{(n)}$ são abertos em $X$

Demonstração. A segunda união, pela proposição anterior, é o complementar de $\overline{X^{(n-1)}}$ e, portanto aberta. A primeira união pode ser escrita como união de dois abertos $\bigcup_{k=0}^{n-3} X^{(k)} \cup X^{(n-1)}$ e $\bigcup_{k=0}^{n-2} X^{(k)} \cup X^{(n)}$

Através desta cobertura disjunta, vamos classificar os pontos conforme sua localização. Para cada $x \in X$ definimos a ordem de acumulação de $x$ como:

$$
r(x)=n, \text { se } x \in X^{(n)}
$$

O espaço será classificado através de um conjunto denominado espectro de acumulação de $X$ :

$$
s(X)=\{r(x): x \in X\}=\left\{n: X^{(n)} \neq \varnothing\right\} .
$$

Exemplos.

1. $s(\{0\} \cup\{1 / n: n \in \mathbb{N}\})=\{0,2\}$

2. $s(\mathcal{C})=\{1\}$

3. $s\left(\mathcal{C}_{2}\right)=\{0,2\}$

4. $s\left(\mathcal{C}_{n}\right)=\{0, \ldots, n-2, n\}$

5. $s\left(\mathcal{C}_{n} \oplus \mathcal{C}_{n-1}\right)=\{0, \ldots, n\}$ 
onde $\mathcal{C}_{-1}=\varnothing, \mathcal{C}_{0}=\{1\}, \mathcal{C}_{1}=\mathcal{C}, \mathcal{F}$ é a família dos intervalos removidos de $\mathcal{C}, \mathcal{C}_{n-2}^{I} \oplus \mathcal{C}_{n-3}^{I}$ é uma cópia de $\mathcal{C}_{n-2} \oplus \mathcal{C}_{n-3}$ dentro de $I$, para $I \in \mathcal{F}$, e

$$
\mathcal{C}_{n}=\mathcal{C} \cup \bigcup_{I \in \mathcal{F}} \mathcal{C}_{n-2}^{I} \oplus \mathcal{C}_{n-3}^{I}
$$

Para mostrarmos a veracidade dos exemplos 4 e 5 vamos utilizar a indução. Os casos $n=0,1,2$ são verificados nos exemplos 1,2 e 3 . Para $n>2$, temos, por hipótese de indução, $s\left(C_{n-3} \oplus C_{n-2}\right)=\{0, \ldots, n-2\}$.

Mas $C_{n}$ possui um subespaço aberto-fechado $C_{n-3} \oplus C_{n-2}$ e portanto, $s\left(C_{n}\right) \supseteq\{0, \ldots, n-2\}$. Ainda mais

$$
C_{n}^{(k)} \supseteq \bigcup_{I \in \mathcal{F}}\left(C_{n-3}^{I} \oplus C_{n-2}^{I}\right)^{(k)}, \text { para todo } k \leq n-2
$$

Por outro lado, a cópia de $\mathcal{C}$ está na aderência do lado direito da inclusão anterior, logo disjunta de $C_{n}^{(k)}$ para $k \leq n-2$, obtendo a igualdade. Então

$$
C_{n}^{(k)}=\bigcup_{I \in \mathcal{F}}\left(C_{n-3}^{I} \oplus C_{n-2}^{I}\right)^{(k)} \text {, para todo } k \leq n-2
$$

e cada $C_{n}^{(k)}(k \leq n-2)$ é denso em $C_{n}$. Portanto,

$$
C_{n}^{(n-1)} \subseteq C_{n} \backslash \overline{C_{n}^{(n-2)}}=\varnothing
$$

já que $C_{n}^{(n-1)} \cap \overline{C_{n}^{(n-2)}}=\varnothing$, e

$$
C_{n}^{(n)}=C_{n} \backslash \bigcup_{I \in \mathcal{F}} C_{n-3}^{I} \oplus C_{n-2}^{I}=\mathcal{C} .
$$

Finalmente temos,

$$
s\left(C_{n}\right)=\{0, \ldots, n-2, n\}
$$

$\mathrm{e}$

$$
s\left(C_{n-1} \oplus C_{n}\right)=s\left(C_{n-1}\right) \cup s\left(C_{n}\right)=\{0, \ldots, n\} .
$$


Algumas observações importantes:

4.8. O Corolário 4.4 nos permite concluir que todos os possiveis espectros finitos são da forma $\{0, \ldots, n\}$ ou $\{0, \ldots, n-2, n\}$ com $n \geq 0$

4.9. Para todo $n \geq 0$, temos $(X \oplus Y)^{(n)}=X^{(n)} \oplus Y^{(n)}$, e conseqüentemente, $s(X \oplus Y)=s(X) \cup s(Y)$.

4.10. Se $X$ é compacto, o único espectro infinito possível é $\mathbb{N} \cup\{\infty\}$.

Demonstração. Suponha $X^{(n)} \neq \varnothing$ para todo $n \in \mathbb{N}$. Seja $\left\{n_{1}, \ldots, n_{p}\right\} \subset \mathbb{N}$ e $m=\max \left\{n_{1}, \ldots, n_{p}\right\}$. Pelo Corolário 4.4, $\overline{X^{(m)}}=X^{(m)} \cup \bigcup_{k \geq m+2} X^{(k)} \mathrm{e}$ com isso, $\bigcup_{k \geq m+2} X^{(k)} \subseteq \overline{X^{\left(n_{1}\right)}} \cap \cdots \cap \overline{X^{\left(n_{p}\right)}}$.

Logo $\left\{\overline{X^{(k)}}: k \in \mathbb{N}\right\}$ tem a propriedade da intersecção finita e como $X$ é compacto,

$$
X^{(\infty)}=\bigcap_{n=0}^{\infty} \overline{X^{(n)}} \neq \varnothing
$$

Obs 4.11. Um exemplo de um espaço em $\mathcal{Z}_{0}$ com espectro infinito é o compactificado de Alexandroff de $\bigoplus_{n=0}^{\infty} \mathcal{C}_{n}$, já que possui pontos de todas as ordens finitas.

Definição 4.12. Um espaço métrico compacto zero-dimensional é pleno (full) se $X^{(n)} \neq \varnothing$ implica $\overline{X^{(n)}}$ é homeomorfo ao conjunto de Cantor para todo inteiro $n \geq 1$.

Alguns fatos:

4.13. Espaços finitos são plenos.

4.14. Soma de dois espaços plenos é um espaço pleno.

4.15. Subconjunto aberto-fechado de um espaço pleno é pleno.

4.16. Todos os $\mathcal{C}_{n}$ são plenos. 
Com estas definições podemos apresentar o próximo teorema que nos permite determinar se dois espaços são homeomorfos, conhecendo seus espectros de acumulação.

Teorema 4.17. Dois espaços plenos com o mesmo espectro de acumulação e a mesma quantidade de pontos isolados são homeomorfos.

Demonstração. As hipóteses do teorema definem uma relação de Vaught que nos permite concluir que os espaços são homeomorfos. Assim, a relação de Vaught será, para $X, Y \in \mathcal{Z}_{0}, X R Y$ se:

\section{i $X$ e $Y$ são plenos}

ii $s(X)=s(Y)$ é finito

iii $X$ e $Y$ possuem a mesma quantidade de pontos isolados

Para verificarmos que é uma relação de Vaught, vamos nos ater a última condição da definição de relação de Vaught, a saber: se $X R\left(\dot{Y}_{0} \oplus Y_{1}\right)$ então existe uma decomposição $X=X_{0} \oplus X_{1}$ tal que $X_{0} R Y_{0}$ e $X_{1} R Y_{1}$. As outras duas condições (simetria e $X R \varnothing \Rightarrow X=\varnothing$ ) são imediatas. Como todo subespaço aberto-fechado de um espaço pleno é pleno, verificamos $i$. Vamos analisar a condição $i i$, pois ao encontrarmos $X_{0}$ e $X_{1}$ que a satisfaçam coṇseguimos facilmente fazer uma troca de abertos-fechados entre $X_{0}$ e $X_{1}$ para satisfazer iii sem afetar $i i$. Podemos assumir que tanto $Y_{0}$ e $Y_{1}$ quanto $X^{(k)}$ (para $k \in s(X)$ ) são infinitos. Sejam $s=s(X), t_{0}=s\left(Y_{0}\right)$ e $t_{1}=s\left(Y_{1}\right)$. Sem perda de generalidade assumimos $\max t_{0} \leq \max t_{1}=\max s=n$.

- Caso 1. $t_{0} \subseteq t_{1}=s$.

Escolhemos $x_{i}, z_{i}$ pontos distintos de $X^{(i)}$ para cada $i \in t_{0}$ (podemos escolher já que assumimos que todos os $X^{(i)}$ são infinitos). Pelo fato 4 sabemos que $\bigcup_{k \in t_{0}} X^{(k)}$ é aberto. Logo, por $X$ ser zero-dimensional, é possível obter abertos-fechados $U_{i}\left(i \in t_{o}\right)$ tais que $x_{i} \in U_{i}$ e $z_{j} \notin U_{i}$ para todo $j \in t_{0}$. Seja $X_{0}=\bigcup_{i \in t_{0}} U_{i}$ e $X_{1}=X \backslash X_{0}$. Note que $X_{0} \cap U_{i}$ 
é não-vazio se $i \in t_{0}$ e $X_{1} \cap U_{i}$ é não vazio para todo $i \in s$. Logo $s\left(X_{0}\right)=t_{0}, s\left(X_{1}\right)=s=t_{1}$.

Observe que sempre que tivermos $s=(0, \ldots, n-2, n)$ estaremos no caso 1 pois como $\max t_{1}=\max s$ não há possibilidade de $t_{1} \varsubsetneqq s$.

- Caso 2. $t_{0}$ e $t_{1}$ são subconjuntos próprios de $s$.

Neste caso necessariamente $s=\{0, \ldots, n\}$. Como max $t_{1}=n$, teremos $t_{0}=\{0, \ldots, n-3, n-1\}$ e $t_{1}=\{0, \ldots, n-2, n\}$.

Pelo fato 4 temos que $X^{(n-2)} \cup X^{(n)}$ e $X^{(n-1)}$ são abertos-fechados relativos em $X^{(n-2)} \cup X^{(n-1)} \cup X^{(n)}$. Com isso escolhemos $X_{0} \subseteq X$ um aberto-fechado tal que

$$
X_{0} \cap\left(X^{(n-2)} \cup X^{(n-1)} \cup X^{(n)}\right)=X^{(n-1)} .
$$

Assim, $s\left(X_{0}\right) \cap\{n-2, n-1, n\}=\{n-1\}$ e, consequentemente $s\left(X_{0}\right)=\{0, \ldots, n-3, n-1\}$. Assim, para $X_{1}=X \backslash X_{0}$, teremos $s\left(X_{1}\right)=t_{1}$.

Obs 4.18. Note que $\{0\} \cup\{1 / n: n \in \mathbb{N}\}$ e o espaço de Pelczynski $\mathcal{C}_{2}$ possuem o mesmo espectro de acumulação $\{0,2\}$ mas não são homeomorfos. Isto mostra que a hipótese no teorema anterior de que os espaços sejam plenos é essencial. 
Agora iremos mostrar um resultado análogo ao teorema anterior para a classe dos espaços métricos zero-dimensionais enumeráveis. Neste caso, não pode ser aplicado o teorema de Vaught já que os espaços não são compactos. A demonstração baseia-se diretamente nos espectros de acumulação dos espaços.

O lema e as definições a seguir serão utilizados na demonstração do teorema.

Lema 4.19. Seja $X$ zero-dimensional com $s(X)=\{0, \ldots, n-1, n\}$. Existe uma decomposição $X=Z \oplus W$, tal que $Z$ e $W$ são abertos-fechados disjuntos $e$
a) $s(Z)=\{0\}$ e $s(W)=\{1\}$, para $n=1$
b) $s(Z)=\{0,2\}$ e $s(W)=\{1\}$, para $n=2$
c) $s(Z)=\{0, \ldots, n-2, n\}$ e $s(W)=\{0, \ldots, n-3, n-1\}$, para $n \geq 3$.

\section{Demonstração.}

a) Imediato já que $X=X^{(0)} \oplus X^{(1)}$.

b) Basta observar que $X^{(0)} \cup X^{(2)}$ e $X^{(1)}$ são abertos.

c) Como $X^{(n-1)}$ e $X^{(n)}$ são fechados disjuntos e $X$ é zero-dimensional, existe um aberto-fechado $U$ tal que $X^{(n)} \subset U$ e $U \cap X^{(n-1)}=\varnothing . \quad$ Logo $S(U)=\{0, \ldots, n-2, n\}$. Seja $V=X \backslash U, \operatorname{temos} S(V)=\{0, \ldots, n-3, n-1\}$ ou $S(V)=\{0, \ldots, n-2, n-1\}$. No primeiro caso $Z=U$ e $W=V$. No segundo caso, se $n=3, s(U)=\{0,1,3\}$ e $s(V)=\{0,2\}$ e também escolhemos $Z=U$ e $W=V$. Para $n>3$ decompomos ainda $V$ em dois abertos-fechados disjuntos $U^{\prime}$ e $V^{\prime}$ tais que $S\left(U^{\prime}\right)=\{0, \ldots, n-3, n-1\}$ e $S\left(V^{\prime}\right)=\{0, \ldots, n-4, n-2\}$ ou $S\left(V^{\prime}\right)=\{0, \ldots, n-3, n-2\}$, e então definimos $Z=U \cup V^{\prime}$ e $W=U^{\prime}$.

Definição 4.20. Um espaço métrico enumerável $X$ é $\mathbb{Q}$-pleno $(\mathbb{Q}$-full), se para todo $n>0, X^{(n)} \neq \varnothing$ implica que $X^{(n)}$ é homeomorfo ao conjunto dos racionais $\mathbb{Q}$.

Definição 4.21. Dois espaços $\mathbb{Q}$-plenos $X$ e $Y$ são equivalentes se possuem o mesmo espectro finito e a mesma quantidade de pontos isolados. 
Proposição 4.22. Sejam $X$ e $Y$ espaços $\mathbb{Q}$-plenos equivalentes tais que $s(X)=s(Y)=\{1\}$ ou $s(X)=s(Y)=\{0, \ldots, n-2, n\}$ para $n \geq 2$. Se $X$ pode ser decomposto em dois abertos-fechados $X^{\prime}$ e $X^{\prime \prime}$, então existe uma decomposição de $Y$ em dois abertos-fechados $Y^{\prime}$ e $Y^{\prime \prime}$ com $X^{\prime}$ equivalente a $Y^{\prime}$ e $X^{\prime \prime}$ equivalente a $Y^{\prime \prime}$.

Demonstração. ver artigo [16]

Teorema 4.23. Se $X$ e $Y$ são equivalentes então são homeomorfos.

Demonstração. Utilizando as enumerações escrevemos $X=\left\{x_{1}, \ldots, x_{i}, \ldots\right\}$ e $Y=\left\{y_{1}, \ldots, y_{i}, \ldots\right\}$ e escolhemos $x_{i_{0}} \in X$ e $y_{i_{0}} \in Y$ os primeiros elementos de máxima ordem de acumulação.

Se $s(X)=s(Y)=\{0, \ldots, n-1, n\}$, devemos primeiramente particionar $X$ em dois subespaços abertos-fechados com espectros $\{0\}$ e $\{1\}$ se $n=1$, $\{0,2\}$ e $\{1\}$ se $n=2$, ou $\{0, \ldots, n-2, n\}$ e $\{0, \ldots, n-3, n-1\}$ para $n>2$. Em seguida, fazemos o mesmo com $Y$ de modo que os seus subespaços tenham a mesma quantidade de pontos isolados que os de $X$ com espectros correspondentes. Já que $X$ é zero-dimensional, podemos observá-lo como subespaço de $\{0,1\}^{\aleph_{0}}$ e dividir cada um dos subespaços de $X$ (ou $X$ mesmo se não houve a primeira decomposição) em dois abertos-fechados com diâmetro menor que $2 / 3$ do diâmetro de $X$. Desta forma, obtemos até 4 subespaços de $X$ com diâmetros reduzidos. Podemos então decompor os subespaços de $Y$ construídos ( $Y$ se não houve a decomposição inicial) em abertos-fechados tais que cada subespaço de $X$ possui um subespaço de $Y$ correspondente com a mesma ordem de acumulação e mesma quantidade de pontos isolados. Agora, para cada subespaço obtido escolhemos o ponto de máxima ordem de acumulação que aparece primeiramente nas enumerações e não foram escolhidos anteriormente. Assim, teremos $x_{i_{1}}, \ldots, x_{i_{k}}$ pontos escolhidos dos subespaços de $X$ e $y_{i_{1}}, \ldots, y_{i_{k}}$, pontos escolhidos dos subespaços de $Y$ correspondentes. Repetimos o processo em cada um dos subespaços de $Y$, e 
assim alternadamente, para garantirmos que o diâmetro dos subespaços de $Y$ também diminuam.

Definimos a função $f: X \mapsto Y$ como $f\left(x_{i_{n}}\right)=y_{i_{n}}$. Para cada $x_{i_{n}}$, obtemos um único $y_{i_{n}}$ correspondente. $\mathrm{E}$, para um ponto $x_{i_{k}} \in X^{(m)}$ ainda não escolhido, como $X^{(0)} \cup \cdots \cup X^{(m)}$ é aberto, em $X$ zero-dimensional, podemos repetir o processo por uma quantidade finita de vezes e encontramos um aberto-fechado $U$ contido em $X^{(0)} \cup \cdots \cup X^{(m)}$ ao qual $x_{i_{k}}$ pertence e tem diâmetro suficientemente pequeno para não encontrar os pontos de ordem $m$ que precedem $x_{i_{k}}$ (na enumeração) e os pontos de ordem maior que $m$ já que $\left\{x_{i_{j}}: x_{i_{j}} \in X^{(m)}\right.$ e $\left.i_{j}<i_{k}\right\} \cup \bigcup_{t=m+1}^{n} X^{(t)}$ é fechado. Portanto $f$ está bem definida. Com o mesmo raciocínio verificamos que $f$ é injetora e sobrejetora. É simples mostrar que $f$ é bicontínua.

Logo $X$ e $Y$ são homeomorfos.

Corolário 4.24 (Sierpinski). Todo espaço métrico enumerável sem pontos isolados é homeomorfo ao conjunto dos racionais $\mathbb{Q}$.

Demonstração. Segue como conseqüência imediata do teorema anterior no caso $s(X)=\{1\}$.

Corolário 4.25. Um espaço compacto não pode ser $\mathbb{Q}$-pleno.

Demonstração. Suponha que $X$ é compacto e $X^{(n)}$ é homeomorfo a $\mathbb{Q}$ para todo $n>0$ que $X^{(n)} \neq \varnothing$. Então $\overline{X^{(n)}}$ é compacto sem pontos isolados e conseqüentemente homeomorfo ao conjunto de Cantor $\mathcal{C}$, o que é impossível já que $X$ é enumerável. 


\section{Capítulo 5}

\section{Espectros de Acumulação em Espaços Exponenciais}

A partir do espectro de acumulação de um espaço métrico compacto zerodimensional $X$ iremos classificar os espectros de acumulação para o espaço exponencial de $X$. Em outras palavras, iremos determinar cada $(\exp (X))^{(i)}$ em função dos $X^{(j)}$.

Para simplificar a notação, denotaremos $(\exp (X))^{(n)} \operatorname{como~} \exp ^{(n)}(X)$. e utilizaremos o seguinte fato que é conseqüência do corolário 4.5

5.1. $X^{(n)}=\complement \overline{X^{(n-1)}} \cap \complement X^{(n-2)} \cap \cdots \cap \complement X^{(0)}$

Vamos generalizar a notação dos conjuntos $\langle A\rangle\rangle A,\left\langle\mathrm{e}\left\langle A_{1}, \ldots, A_{n}\right\rangle\right.$ para $A, A_{1}, \ldots, A_{n}$ subconjuntos quaisquer de $X$ e assim obter uma expressão mais simplificada para cada $\exp ^{(n)}(X)$ :

$$
\begin{aligned}
\langle A\rangle & =\{F \in \exp (X): F \subseteq A\} \\
\rangle A\langle & =\{F \in \exp (X): F \cap A \neq \varnothing\} \\
\left\langle A_{1}, \ldots, A_{n}\right\rangle & =\left\{F \in \exp (X): F \subseteq \bigcup_{i=1}^{n} A_{i} \text { e } F \cap A_{i} \neq \varnothing \text { para } 1 \leq i \leq n\right\}
\end{aligned}
$$


Vamos destacar alguns fatos. Para $A \subset X$, temos

5.2. $\overline{\langle A\rangle}=\langle\bar{A}\rangle$.

\section{Demonstração.}

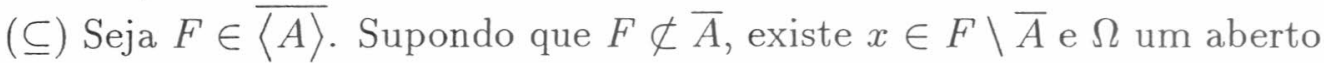
tal que $x \in \Omega$ e $\Omega \cap A=\varnothing$. Mas $\langle X, \Omega\rangle$ é uma vizinhança de $F$ e deve interceptar $\langle A\rangle$, ou seja, existe $K \subset A$ tal que $K \cap \Omega \neq \varnothing$. (absurdo!).

() Por outro lado seja $F \in\langle\bar{A}\rangle$ e $\left\langle U_{1}, \ldots, U_{n}\right\rangle$ um aberto em $\exp (X)$ $\operatorname{com} F \in\left\langle U_{1}, \ldots, U_{n}\right\rangle$. Como $F \subseteq \bar{A}$ e $F \cap U_{i} \neq \varnothing$ para cada $1 \leq i \leq n$, então existe $x_{i} \in A \cap U_{i}$. Logo $\left\{x_{1}, \ldots, x_{n}\right\} \subseteq A \cap \bigcup_{i=1}^{n} U_{i} \neq \varnothing$ e conseqüentemente $\left\{x_{1}, \ldots, x_{n}\right\} \in\langle A\rangle \cap\left\langle U_{1}, \ldots, U_{n}\right\rangle$. Logo $F \in \overline{\langle A\rangle}$.

5.3. $\overline{\rangle A\langle}=\rangle \bar{A}\langle$.

Demonstração. Análoga à demonstração anterior.

5.4. Se $B \subset A \subset X$, então $\overline{\langle A\rangle \cap\rangle B\langle}=\langle\bar{A}\rangle \cap\rangle \bar{B}\langle$.

Demonstração.

$(\subseteq)$ Imediato.

() Seja $B \neq \varnothing$ e $F \in\langle\bar{A}\rangle \cap\rangle \bar{B}\langle$. Temos $F \subseteq \bar{A}$ e $F \cap \bar{B} \neq \varnothing$. Supondo

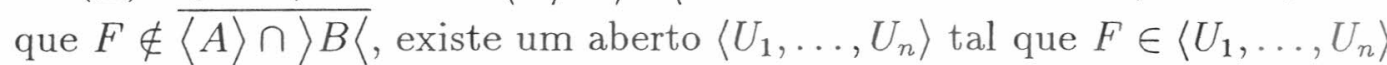
$\mathrm{e}\langle A\rangle \cap\rangle B\left\langle\cap\left\langle U_{1}, \ldots, U_{n}\right\rangle=\varnothing\right.$. Seja $d_{i} \in F \cap U_{i}$ para todo $i$. Logo $d_{i} \in \bar{A}$ e conseqüentemente $U_{i} \cap A \neq \varnothing$. Escolhemos $a_{i} \in U_{i} \cap A$. Como $F \cap \bar{B} \neq \varnothing$, existe $b \in U_{k} \cap B$ para algum $1 \leq k \leq n$. Dado que $B \subset A$, obtemos $\left\{a_{1}, \ldots, a_{n}\right\} \cup\{b\}$ um ponto pertencente a $\left.\left\langle U_{1}, \ldots, U_{n}\right\rangle \cap\langle A\rangle \cap\right\rangle B\langle$ e portanto $F \in \overline{\langle A\rangle \cap\rangle B\langle\text {. }}$

A partir de agora, iremos determinar cada $\exp ^{(n)}(X)$. 
Proposição 5.5. $\exp ^{(0)}(X)=\left\langle X^{(0)}\right\rangle$.

Demonstração. Como $X$ é compacto, todo ponto isolado em $\exp (X)$ é um subconjunto finito de pontos isolados de $X$ (veja proposição 2.7 e obs 2.8).

Proposição 5.6. $\left.\exp ^{(1)}(X)=\right\rangle X^{(1)}\langle$.

Demonstração. Utilizando a definição, temos

$$
\begin{aligned}
& \quad \exp ^{(1)}(X)=\exp (X) \backslash \overline{\exp ^{(0)}}=\exp (X) \backslash \overline{\left\langle X^{(0)}\right\rangle}=\exp (X) \backslash\left\langle\overline{X^{(0)}}\right\rangle= \\
& \rangle X \backslash \overline{X^{(0)}}\langle=\rangle X^{(1)}\langle.
\end{aligned}
$$

Proposição 5.7. $\left.\exp ^{(2)}(X)=\left\langle X^{(0)} \cup X^{(2)}\right\rangle \cap\right\rangle X^{(2)}\langle$

Demonstração. Basta utilizar o fato 5.1 e as proposições anteriores,

$$
\begin{aligned}
\exp ^{(2)}(X) & =\complement \overline{\exp ^{(1)}(X)} \cap \complement_{\exp ^{(0)}(X)} \\
& =\left\langle\complement \overline{X^{(1)}}\right\rangle \cap \complement X^{(0)}\langle \\
& \left.=\left\langle X^{(0)} \cup X^{(2)}\right\rangle \cap\right\rangle \complement X^{(0)}\langle(\text { proposição 4.2) } \\
& \left.=\left\langle X^{(0)} \cup X^{(2)}\right\rangle \cap\right\rangle X^{(2)}\langle
\end{aligned}
$$

Proposição 5.8. $\left.\exp ^{(3)}(X)=\left\langle X^{(0)} \cup X^{(3)}\right\rangle \cap\right\rangle X^{(3)}\langle$

Demonstração. Utilizando o fato 5.1 e as proposições anteriores,

$$
\begin{aligned}
\exp ^{(3)}(X) & =\complement \overline{\exp ^{(2)}(X)} \cap \complement \exp ^{(1)}(X) \cap \complement_{\exp ^{(0)}(X)} \\
& \left.=\complement\left\langle\overline{X^{(0)}}, \overline{X^{(2)}}\right\rangle \cap \complement\right\rangle X^{(1)}\left\langle\cap \complement\left\langle X^{(0)}\right\rangle\right. \\
& =\complement\left\langle\overline{X^{(0)}}, \overline{X^{(2)}}\right\rangle \cap\left\langle\overline{X^{(0)}}\right\rangle \cap \complement\left\langle X^{(0)}\right\rangle \\
& \left.=\left\langle X^{(0)} \cup X^{(3)}\right\rangle \cap\right\rangle X^{(3)}\langle
\end{aligned}
$$


Proposição 5.9. $\left.\exp ^{(4)}(X)=\left\langle X^{(0)} \cup X^{(2)} \cup X^{(4)}\right\rangle \cap\right\rangle X^{(4)}\langle$

Demonstração. Novamente através do fato 5.1 e dos $\exp ^{(n)}(X)$ já calculados

$$
\begin{aligned}
\exp ^{(4)}(X) & =\complement \overline{\exp ^{(3)}(X)} \cap \complement \exp ^{(2)}(X) \cap \complement \exp ^{(1)}(X) \cap \complement \exp ^{(0)}(X) \\
& \left.=\complement\left\langle\overline{X^{(0)}}, \overline{X^{(3)}}\right\rangle \cap \complement\left(\left\langle X^{(0)} \cup X^{(2)}\right\rangle \cap\right\rangle X^{(2)}\langle) \cap \complement\right\rangle X^{(1)}\left\langle\cap \complement\left\langle X^{(0)}\right\rangle\right. \\
& \left.=\left\langle\overline{X^{(0)}}\right\rangle \cap \complement\right\rangle \overline{X^{(3)}}\left\langle\cap \complement\left\langle X^{(0)} \cup X^{(2)}\right\rangle\right. \\
& \left.=\left\langle X^{(0)} \cup X^{(2)} \cup X^{(4)}\right\rangle \cap\right\rangle X^{(4)}\langle
\end{aligned}
$$

Proposição 5.10. $\left.\exp ^{(5)}(X)=\left\langle X^{(0)} \cup X^{(2)} \cup X^{(3)} \cup X^{(5)}\right\rangle \cap\right\rangle X^{(2)} \cup X^{(5)}\langle\cap$ \rangle $X^{(3)} \cup X^{(5)}<$

Demonstração. Com o fato 5.1 e proposições anteriores, obtemos

$$
\begin{aligned}
\exp ^{(5)}(X)= & \complement \overline{\exp ^{(4)}(X)} \cap \complement \exp ^{(3)}(X) \cap \complement \exp ^{(2)}(X) \cap \complement \exp ^{(1)}(X) \cap \complement \exp ^{(0)}(X) \\
= & \complement\left\langle\overline{X^{(0)}}, \overline{X^{(4)}}\right\rangle \cap \complement\left(\left\langle X^{(0)}, X^{(3)}\right\rangle \cap\right\rangle X^{(3)}\langle) \cap \complement\left(\left\langle X^{(0)} \cup X^{(2)}\right\rangle \cap\right\rangle X^{(2)}\langle) \cap \\
& \cap \complement\rangle X^{(1)}\left\langle\cap \complement\left\langle X^{(0)}\right\rangle\right. \\
= & \left.\left\langle\overline{X^{(0)}}\right\rangle \cap\right\rangle \overline{X^{(4)}}\left\langle\cap \complement\left(\left\langle X^{(0)} \cup X^{(3)}\right\rangle \cap\right\rangle X^{(3)}\langle) \cap \complement\left(\left\langle X^{(0)} \cup X^{(2)}\right\rangle \cap\right\rangle X^{(2)}\langle) \cap\right. \\
& \cap \complement\left\langle X^{(0)}\right\rangle \\
= & \left\langle X^{(0)} \cup X^{(2)} \cup X^{(3)} \cup X^{(5)}\right\rangle \cup \complement\left(X^{(0)} \cup X^{(3)}\right\rangle \cap \complement\left(X^{(0)} \cup X^{(2)}\right\rangle \\
= & \left.\left\langle X^{(0)} \cup X^{(2)} \cup X^{(3)} \cup X^{(5)}\right\rangle \cap\right\rangle X^{(2)} \cup X^{(5)}\langle\cap\rangle X^{(3)} \cup X^{(5)}\langle
\end{aligned}
$$


Proposição 5.11. $\exp ^{(6)}(X)=\varnothing$ e conseqüentemente $\exp ^{(n)}(X)=\varnothing$ para todo $n \geq 8$.

Demonstração.

$$
\begin{aligned}
& \exp ^{(6)}(X)=\overline{\complement \exp ^{(5)}(X)} \cap \complement \exp ^{(4)}(X) \cap \operatorname{Cxp}^{(3)}(X) \cap \complement \exp ^{(2)}(X) \cap \complement \exp ^{(1)}(X) \cap \complement \exp ^{(0)}( \\
& =\complement\left\langle\overline{X^{(0)}}, \overline{X^{(2)}}, \overline{X^{(3)}}\right\rangle \cap \complement\left(\left\langle X^{(0)} \cup X^{(2)} \cup X^{(4)}\right\rangle \cap\right\rangle X^{(4)}\langle) \cap \\
& \left.\cap \complement\left(\left\langle X^{(0)}, X^{(3)}\right\rangle \cap\right\rangle X^{(3)}\langle) \cap \complement\left(\left\langle X^{(0)} \cup X^{(2)}\right\rangle \cap\right\rangle X^{(2)}\langle) \cap \complement\right\rangle X^{(1)}\left\langle\cap \complement\left\langle X^{(0)}\right\rangle\right. \\
& \subseteq\left(\left\langle X^{(0)} \cup X^{(3)}\right\rangle \cap \cap \complement\left(\left\langle X^{(0)}, X^{(3)}\right\rangle \cap\right\rangle X^{(3)}\langle) \complement\left\langle X^{(0)}\right\rangle\right) \cup \\
& \cup\left(\left\langle X^{(0)} \cup X^{(2)} \cup X^{(4)}\right\rangle \complement\left(\left\langle X^{(0)} \cup X^{(2)} \cup X^{(4)}\right\rangle \cap\right\rangle X^{(4)}\langle) \cap\right. \\
& \left.\cap \complement\left(\left\langle X^{(0)} \cup X^{(2)}\right\rangle \cap\right\rangle X^{(2)}\langle) \cap \complement\left\langle X^{(0)}\right\rangle\right) \\
& =\varnothing \cup \varnothing
\end{aligned}
$$

Proposição 5.12. $\exp ^{(7)}(X)=\left\langle\overline{X^{(0)}}, \overline{X^{(3)}}, \overline{X^{(4)}}\right\rangle$

Demonstração. De forma similar as demonstrações anteriores,temos

$$
\begin{aligned}
\exp ^{(7)}(X)= & \complement \overline{\exp ^{(6)}(X)} \cap \operatorname{Cexp}^{(5)}(X) \cap \operatorname{Cexp}^{(4)}(X) \cap \complement_{\exp ^{(3)}}(X) \cap \\
& \complement \exp ^{(2)}(X) \cap \exp ^{(1)}(X) \cap \exp ^{(0)}(X) \\
= & \complement\left(\left\langle X^{(0)} \cup X^{(2)} \cup X^{(3)} \cup X^{(5)}\right\rangle \cap\right\rangle X^{(2)} \cup X^{(5)}\langle\cap\rangle X^{(3)} \cup X^{(5)}\langle) \cap \\
& \cap \complement\left(\left\langle X^{(0)} \cup X^{(2)} \cup X^{(4)}\right\rangle \cap\right\rangle X^{(4)}\langle) \cap \\
& \cap \complement\left(\left\langle X^{(0)}, X^{(3)}\right\rangle \cap\right\rangle X^{(3)}\langle) \cap \complement\left(\left\langle X^{(0)} \cup X^{(2)}\right\rangle \cap\right\rangle X^{(2)}\langle) \cap \\
& \cap \complement\rangle X^{(1)}\left\langle\cap \complement\left\langle X^{(0)}\right\rangle\right.
\end{aligned}
$$

Vamos dividir o problema em duas parte. Na primeira calculamos

$$
\begin{aligned}
& \left\langle\overline{X^{(0)}}\right\rangle \cap \complement\left\langle X^{(0)}\right\rangle \cap \complement\left(\left\langle X^{(0)} \cup X^{(2)}\right\rangle \cap\right\rangle X^{(2)}\langle) \cap \complement\left(\left\langle X^{(0)} \cup X^{(2)} \cup X^{(4)}\right\rangle \cap\right\rangle X^{(4)}\langle)= \\
= & \left.\left\langle\overline{X^{(0)}}\right\rangle \cap\right\rangle X^{(3)} \cap \bigcup_{n \geq 5} X^{(n)}\langle= \\
= & \left.\left\langle\overline{X^{(0)}}\right\rangle \cap\right\rangle \overline{X^{(3)}}\langle
\end{aligned}
$$


E, na segunda,

$$
\begin{aligned}
& \left\langle\overline{X^{(0)}}\right\rangle \cap \complement\left\langle X^{(0)}\right\rangle \cap \complement\left(\left\langle X^{(0)} \cup X^{(2)}\right\rangle \cap\right\rangle X^{(2)}\langle) \cap \complement\left(\left\langle X^{(0)} \cup X^{(3)}\right\rangle \cap\right\rangle X^{(3)}\langle) \cap \\
& \cap \complement\left(\left\langle X^{(0)} \cup X^{(2)} \cup X^{(3)} \cup X^{(5)}\right\rangle \cap\right\rangle X^{(2)} \cup X^{(5)}\langle\cap\rangle X^{(3)} \cup X^{(5)}\langle)= \\
= & \left.\left\langle\overline{X^{(0)}}\right\rangle \cap\right\rangle X^{(4)} \cap \bigcup_{n \geq 6} X^{(n)}\langle= \\
= & \left.\left\langle\overline{X^{(0)}}\right\rangle \cap\right\rangle \overline{X^{(4)}}\langle
\end{aligned}
$$

E assim temos

$$
\left.\left\langle\overline{X^{(0)}}\right\rangle \cap\right\rangle \overline{X^{(3)}}\langle\cap\rangle \overline{X^{(4)}}\left\langle=\left\langle\overline{X^{(0)}}, \overline{X^{(3)}}, \overline{X^{(4)}}\right\rangle\right.
$$

Proposição 5.13. Para todo inteiro positivo $n, \overline{\exp ^{(n)}(X)}$ é vazio ou homeomorfo ao conjunto de Cantor.

Demonstração. Primeiramente calculamos a aderência de cada conjunto determinado nas proposições anteriores deste capítulo.

$$
\begin{aligned}
& \overline{\exp ^{(1)}(X)}=\overline{X^{(1)}}\langle \\
& \overline{\exp ^{(2)}(X)}=\left\langle\overline{X^{(0)}}, \overline{X^{(2)}}\right\rangle \\
& \overline{\exp ^{(3)}(X)}=\left\langle\overline{X^{(0)}}, \overline{X^{(3)}}\right\rangle \\
& \overline{\exp ^{(4)}(X)}=\left\langle\overline{X^{(0)}}, \overline{X^{(4)}}\right\rangle \\
& \overline{\exp ^{(5)}(X)}=\left\langle\overline{X^{(0)}}, \overline{X^{(2)}}, \overline{X^{(3)}}\right\rangle \\
& \overline{\exp ^{(7)}(X)}=\left\langle\overline{X^{(0)}}, \overline{X^{(3)}}, \overline{X^{(4)}}\right\rangle \\
& \overline{\exp ^{(n)}(X)}=\varnothing \text { para } n=6 \text { ou } n \geq 8
\end{aligned}
$$

De acordo com o corolário 3.3 sabemos que um espaço métrico compacto zero-dimensional não vazio é homeomorfo ao conjunto de Cantor se não possui pontos isolados. Vamos, então, verificar este fato para cada conjunto $\overline{\exp ^{(n)}(X)}$. 
Para $n>1$, para todo $F \in \overline{\exp ^{(n)}(X)}$ teremos que $F \subset \overline{X^{(0)}}$ e deve existir um ponto $x \in F \backslash X^{(0)}$. Pegamos uma seqüência $\left(x_{k}\right)_{k \in \mathbb{N}} \subseteq X^{(0)}$ que converge para $x$ e definimos $F_{k}=F \cup\left\{x_{k}\right\}$ se $x_{k} \notin F$ e $F_{k}=F \backslash\left\{x_{k}\right\}$ se $x_{k} \in F$. Como cada $x_{k}$ é isolado, o respectivo $F_{k}$ é fechado e assim, temos a seqüência $\left(F_{k}\right)_{k \in \mathbb{N}}$ que converge para $F$. Como cada $F_{k}$ difere de $F$ em apenas um ponto de $X^{(0)}, F_{k}$ intercepta todo $\overline{X^{(m)}}$ para $m \geq 2$ que $F$ intercepta. Logo $F_{k}$ pertence a $\overline{\exp ^{(n)}(X)}$.

Para $n=1$. Seja $x \in F \cap \overline{X^{(1)}}$ e $\left(x_{k}\right)_{k \in \mathbb{N}} \subseteq X^{(1)} \backslash\{x\}$ uma seqüência que converge para $x$. Para cada $k$ escolhemos um aberto-fechado $U_{k} \subset X^{(1)}$ tal que $x_{k} \in U_{k}$ e $\delta\left(U_{k}\right)<d\left(x, x_{k}\right) / 3^{k}$ e definimos $F_{k}=F \cup U_{k}$ se $U_{k} \nsubseteq F$ ou $F_{k}=F \backslash U_{k}$ caso contrário. Assim, $F_{k} \rightarrow F$ e como $x$ pertence a todo $F_{k}$, $\left.F_{k} \in\right\rangle \overline{X^{(1)}}\left\langle\right.$ e conseqüentemente $F$ não é isolado em $\overline{\exp ^{(n)}(X)}$.

A seguir apresentaremos uma tabela que relaciona os possíveis espectros de um espaço em $\mathcal{Z}_{0}$ e o respectivo espectro de seu exponencial. Como o teorema anterior garante que todo espaço exponencial de um espaço métrico compacto zero-dimensional é pleno, determinaremos os espaços exponencialmente completos bastando observar seus pontos isolados e seu espectro de acumulação (teorema 4.17).

Observe que se a quantidade dos pontos isolados de um espaço $X$ é finita, a cardinalidade do conjunto dos pontos isolados de $\exp (X)$ é $2^{\left|X^{(0)}\right|}-1$ já que é formado pelo conjunto das partes não vazias de $X^{(0)}$. Isto nos leva a concluir que os espaços exponencialmente completos ou não possuem pontos isolados, ou a quantidade de pontos isolados é infinita. 
Lista DE TODOS OS EXPONENCIAIS EM $\mathcal{Z}_{0}$

\begin{tabular}{|r|l|l|l|}
\hline & $s(X)$ & $s(\exp (X))$ & $\exp (X)$ \\
\hline 1 & $\{0\}$ & $\{0\}$ & $\left\{2,3, \ldots, 2^{\left|X^{(0)}\right|}\right\}$ \\
2 & $\{1\}$ & $\{1\}$ & $\mathcal{C}_{1}$ \\
3 & $\{0,1\}$ & $\{0,1\}$ & $\mathcal{C}_{1} \cup\left\{2,3, \ldots, 2^{\left|X^{(0)}\right|}\right\}$ \\
4 & $\{0,2\}$ & $\{0,2\}$ & $\mathcal{C}_{2}$ \\
5 & $\{0,1,2\}$ & $\{0,1,2\}$ & $\mathcal{C}_{1} \oplus \mathcal{C}_{2}$ \\
6 & $\{0,1,3\}$ & $\{0,1,3\}$ & $\mathcal{C}_{3}$ \\
7 & $\{0,1,2,3\}$ & $\{0,1,2,3,5\}$ & $\mathcal{C}_{5}$ \\
8 & $\{0,1,2,4\}$ & $\{0,1,2,4\}$ & $\mathcal{C}_{4}$ \\
9 & $\{0,1,2,3,4\}$ & $\{0,1,2,3,4,5,7\}$ & $\mathcal{C}_{7}$ \\
10 & $\{0,1,2,3,5\}$ & $\{0,1,2,3,5\}$ & $\mathcal{C}_{5}$ \\
11 & $\{0,1,2,3,4,5\}$ & $\{0,1,2,3,4,5,7\}$ & $\mathcal{C}_{7}$ \\
12 & demais espectros & $\{0,1,2,3,4,5,7\}$ & $\mathcal{C}_{7}$ \\
\hline
\end{tabular}

Através desta tabela podemos concluir os seguintes resultados:

Teorema 5.14 (Marjanović). Os únicos espaços exponenciais a menos de um homeomorfismo na classe dos espaços métricos compactos zero-dimensionais são: $\left\{2,3, \ldots, 2^{n}\right\}$ para $n \in \mathbb{N}^{*}, \mathcal{C}_{1} \cup\left\{2,3, \ldots, 2^{n}\right\}$ paran $\in \mathbb{N}^{*}, \mathcal{C}_{1}, \mathcal{C}_{2}, \mathcal{C}_{1} \oplus \mathcal{C}_{2}$, $\mathcal{C}_{3}, \mathcal{C}_{4}, \mathcal{C}_{5}$ e $\mathcal{C}_{7}$

Corolário 5.15. As 9 soluções da equação $X=\exp (X)$ na classe dos espaços métricos compactos zero-dimensionais não triviais são: $\mathcal{C}_{0}, \mathcal{C}_{1}, \mathcal{C}_{0} \oplus \mathcal{C}_{1}$, $\mathcal{C}_{2}, \mathcal{C}_{1} \oplus \mathcal{C}_{2}, \mathcal{C}_{3}, \mathcal{C}_{4}, \mathcal{C}_{5}, \mathcal{C}_{7}$ 


\section{Apêndice A}

\section{Exponenciais de outros espaços topológicos}

Este apêndice tem por finalidade expor alguns exemplos de exponenciais de espaços diferentes dos que estávamos trabalhando. A idéia é estabelecer se os espaços são exponencialmente completos ou não.

O primeiro espaço que veremos é $\{0,1\}^{\aleph_{1}}$, um espaço zero-dimensional compacto mas não metrizável. É um exemplo de espaço exponencialmente completo, fato mostrado por S.Sirota [12] em 1968. 


\section{A.1 $\{0,1\}^{\aleph_{1}}$}

Para determinarmos o exponencial de $\{0,1\}^{\aleph_{1}}$ vamos utilizar a seguinte construção:

Para cada $\alpha \in \aleph_{1} \backslash\{0\}$, seja $X_{\alpha}$ o produto topológico $\left(\{0,1\}^{\aleph_{0}}\right)^{\alpha}$ e para cada $0<\alpha<\beta<\aleph_{1}$ seja

$$
\begin{array}{ll}
\Pi_{\alpha}^{\beta}: & X_{\beta} \longrightarrow X_{\alpha} \\
\Pi_{\alpha}^{\beta}: & \mathcal{C}^{\beta} \longrightarrow \mathcal{C}^{\alpha} \quad \text { projeção contínua e aberta. }
\end{array}
$$

Se $\beta>\alpha>\gamma>0$, então

$$
\Pi_{\gamma}^{\alpha} \circ \Pi_{\alpha}^{\beta}=\Pi_{\gamma}^{\beta}
$$

Estas condições determinam que $\left\{X_{\beta} ; \Pi_{\alpha}^{\beta} ; \aleph_{1} \backslash\{0\}\right\}$ é um sistema inverso e vale:

$$
\exp \left(\lim _{\leftarrow} X_{\beta}\right)=\lim _{\leftarrow} \exp \left(X_{\beta}\right)
$$

Além disso, $\lim _{\leftarrow} X_{\beta}$ é homeomorfo a $\{0,1\}^{\aleph_{1}}$.

Para cada $0 \leq \beta<\aleph_{1}, X_{\beta}$ é homeomorfo a $\mathcal{C}$ e, também, $\exp \left(X_{\beta}\right)$ é homeomorfo a $\mathcal{C}$. $\operatorname{Logo} \exp \left(\{0,1\}^{\aleph_{1}}\right)$ é homeomorfo a $\{0,1\}^{\aleph_{1}}$. Trata-se de um espaço exponencialmente completo.

\section{A.2 $\{0,1\}^{\aleph_{2}}$}

Em 1976 Shapiro publicou um artigo [10] em que, também utilizando limites inversos e uma construção complexa, mostrou que $\{0,1\}^{\aleph_{2}}$ não é exponencialmente completo. O espaço em questão não é metrizável e é zerodimensional compacto. 


\section{A.3 $[0,1]$}

Desde a década de 20 a escola polonesa de topologia conjecturava que $\exp ([0,1])$ fosse homeomorfo ao cubo de Hilbert $[0,1]^{\aleph_{0}}$. Em 1975 Schori e West mostraram a veracidade desta afirmação concluindo-se que $[0,1]$ não é exponencialmente completo. Este espaço é metrizável, compacto mas não é zero-dimensional. 


\section{Referências Bibliográficas}

[1] R. Engelking, General topology, Heldernmann, 1989.

[2] J. G. Hocking and G. S. Young, Topology, Addison-Wesley, 1961.

[3] K. Kunen, Set theory: independence proofs, North Holland, 1980.

[4] M. M. Marjanović, Exponentially complete spaces III, Publications de l'Institute Mathématique 14 (1972), no. 28, 97-109.

[5] M. M. Marjanović and A. R. Vučemilović, Two non-homeomorphic countable spaces having homeomorphic squares, Comm. Math. Univ. Caroline 26 (1985), 579-588.

[6] E. Michael, Topologies on spaces of subsets, Trans. Amer. Math. Soc. 71 (1951), 152-182.

[7] Jr. S. B. Nadler, Hyperspaces of sets, Marcel Dekker, 1978.

[8] A. Pelczynski, A remark on space $2^{X}$ for zero-dimentional X, Bull. Acad. Polon. Sci. Ser. Math. 13 (1965), 85-89.

[9] R. M. Schori and J. E. West, The hyperspace of the closed unit interval is a Hilbert cube, Transactions of the American Mathematical Society 213 (1975), 217-235.

[10] L. Shapiro, The space of closed subsets of $D^{\aleph_{2}}$ is not a dyadic bicompact, Soviet Math. Dokl. 17 (1976), 937-941. 
[11] G. F. Simmons, Introduction to topology and modern analysis, McGrawHill, 1963.

[12] S. Sirota, The spectral representation of spaces of closed subsets of bicompacta, Soviet Math. Dokl. 9 (1968), 997-1000.

[13] S. Todorcević, Topics in topology, Lecture Notes in Mathematics, vol. 1652, Springer, 1997.

[14] H. Torunczyk, Characterizing Hilbert space topology, Fund. Math. (1981), 247-262.

[15] Jan van Mill, Characterization of some zero-dimensional separable metric spaces, Transactions of the American Mathematical Society $\mathbf{1 . 2 6 4}$ (1981), 205-215.

[16] A. R. Vučemilović, On countable spaces, Mathematica Balkanica 4.127 (1974), 579-588.

[17] A. N. Vybornov, Exponentials of zero-dimensional Polish spaces, Soviet Math. Dokl. 32 (1985), 532-536. 


\section{Índice Remissivo}

$2^{X}, 12$

$X^{(n)}, 24$

$X^{(\infty)}, 24$

[01], 45

$[X]^{\leq n}, 13$

C, 9

$\mathcal{C}_{2}, 20$

$\mathcal{C}_{n}, 28$

$\langle U\rangle, 12,36$

$\left\langle U_{1}, \ldots, U_{n}\right\rangle, 12,36$

\rangle $U\langle, 12,36$

$\bigoplus_{s \in S} X_{s}, 10$

$\delta(A), 11$

$\exp (X), 12$

$\exp ^{(n)}(X), 35$

$\{0,1\}^{\aleph_{1}}, 44$

$\{0,1\}^{\aleph_{2}}, 44$

$r(x), 27$

$s(X), 27$

$\mathcal{Z}_{0}, 17$

conjunto

de Cantor, 9, 20

diâmetro, 11

dos racionais, 34

diâmetro de um conjunto, 11

enésima potência simétrica, 13

espaço

exponencialmente completo, 16

de Pelczynski, 20

exponencial, 12

espectro de acumulação, 35

ponto de acumulação, 16

ponto isolado, 16

metrizável, 10

pleno, 29

Q-pleno, 32

zero-dimensional, 7

espaços

equivalentes, 32,33

espectro de acumulação, 27, 30

espaço exponencial, 35

finito, 29

infinito, 29

full space, 29

hiper-espaço, 12

ordem de acumulação, 27

relação de Vaught, 17, 20, 30 
soma topológica, 10

teorema

Cantor/Brouwer, 20

Homeomorfismo de Vaught, 17

Intersecção de Cantor, 11

Marjanović, 42

Pelczynski, 20

Redução, 8

Separação, 8

Sierpinski, 34

topologia

de Vietoris, 13

finita, 13 